\title{
EGFR phosphorylation of DCBLD2 recruits TRAF6 and stimulates AKT-promoted tumorigenesis
}

\author{
Haizhong Feng, ${ }^{1,2}$ Giselle Y. Lopez, ${ }^{3}$ Chung Kwon Kim, ${ }^{2}$ Angel Alvarez, ${ }^{2}$ Christopher C. Duncan, ${ }^{3}$ Ryo Nishikawa, ${ }^{4}$ Motoo Nagane, ${ }^{5}$ \\ An-Jey A. Su, ${ }^{6}$ Philip E. Auron, ${ }^{6}$ Matthew L. Hedberg,' Lin Wang, ${ }^{7}$ Jeffery J. Raizer, ${ }^{2}$ John A. Kessler, ${ }^{2}$ Andrew T. Parsa, ${ }^{8}$ \\ Wei-Qiang Gao, ${ }^{1}$ Sung-Hak Kim, ${ }^{9}$ Mutsuko Minata, ${ }^{9}$ Ichiro Nakano, ${ }^{9}$ Jennifer R. Grandis, ${ }^{7}$ Roger E. McLendon, ${ }^{3}$ \\ Darell D. Bigner, ${ }^{3}$ Hui-Kuan Lin, ${ }^{10}$ Frank B. Furnari, ${ }^{11}$ Webster K. Cavenee, ${ }^{11}$ Bo Hu, ${ }^{2}$ Hai Yan, ${ }^{3}$ and Shi-Yuan Cheng ${ }^{1,2}$ \\ 'State Key Laboratory of Oncogenes and Related Genes, Renji-Med X Clinical Stem Cell Research Center, Ren Ji Hospital, School of Medicine, Shanghai Jiao Tong University, Shanghai, China. \\ 2Department of Neurology and Northwestern Brain Tumor Institute, Center for Genetic Medicine, Robert H. Lurie Comprehensive Cancer Center, Northwestern University Feinberg School of Medicine, \\ Chicago, Illinois, USA. PPediatric Brain Tumor Foundation Institute at Duke, The Preston Robert Tisch Brain Tumor Center, and Department of Pathology, Duke University Medical Center, Durham, \\ North Carolina, USA. ${ }^{4}$ Department of Neuro-Oncology/Neurosurgery, International Medical Center, Saitama Medical University, Saitama, Japan. ${ }^{5}$ Department of Neurosurgery, Kyorin University, Tokyo, \\ Japan. ${ }^{6}$ Department of Biological Sciences, Duquesne University, Pittsburgh, Pennsylvania, USA. Departments of Otolaryngology and Pharmacology and Chemical Biology, University of Pittsburgh \\ School of Medicine, Pittsburgh, Pennsylvania, USA. ${ }^{8}$ Department of Neurological Surgery and Northwestern Brain Tumor Institute, Robert H. Lurie Comprehensive Cancer Center, Northwestern University \\ Feinberg School of Medicine, Chicago, Illinois, USA. ${ }^{9}$ Department of Neurological Surgery, James Comprehensive Cancer Center, The Ohio State University, Columbus, Ohio, USA. ${ }^{10}$ Department of Molecular \\ and Cellular Oncology, University of Texas MD Anderson Cancer Center, Houston, Texas, USA. "'Ludwig Institute for Cancer Research and UCSD, School of Medicine, La Jolla, California, USA.
}

\begin{abstract}
Aberrant activation of EGFR in human cancers promotes tumorigenesis through stimulation of AKT signaling. Here, we determined that the discoidina neuropilin-like membrane protein DCBLD2 is upregulated in clinical specimens of glioblastomas and head and neck cancers (HNCs) and is required for ECFR-stimulated tumorigenesis. In multiple cancer cell lines, EGFR activated phosphorylation of tyrosine 750 (Y750) of DCBLD2, which is located within a recently identified binding motif for TNF receptor-associated factor 6 (TRAF6). Consequently, phosphorylation of DCBLD2 Y750 recruited TRAF6, leading to increased TRAF6 E3 ubiquitin ligase activity and subsequent activation of AKT, thereby enhancing EGFR-driven tumorigenesis. Moreover, evaluation of patient samples of gliomas and HNCs revealed an association among ECFR activation, DCBLD2 phosphorylation, and poor prognoses. Together, our findings uncover a pathway in which DCBLD2 functions as a signal relay for oncogenic EGFR signaling to promote tumorigenesis and suggest DCBLD2 and TRAF6 as potential therapeutic targets for human cancers that are associated with EGFR activation.
\end{abstract}

\section{Introduction}

A hallmark of human cancers is that oncogenic signaling stimulated by amplified and overexpressed genes is aberrantly active (1). In human glioblastoma (GBM) and head and neck cancer (HNC), EGFR is frequently amplified and often co-overexpressed with a constitutively active mutant, EGFRvIII (also referred to as $\triangle$ EGFR and de2-7EGFR) $(2,3)$. EGFR is also commonly overexpressed and mutated in lung cancers (4). The activated oncogenic EGFR signaling in these cancers contributes to cancer development, progression, and resistance to current therapies (4-6). Mechanistically, EGFR drives tumorigenesis primarily through activation of AKT signaling, thereby stimulating cancer cell proliferation, survival, and drug resistance. In human GBM

\footnotetext{
Authorship note: Haizhong Feng and Giselle Y. Lopez contributed equally to this work. Note regarding evaluation of this manuscript: Manuscripts authored by scientists associated with Duke University, The University of North Carolina at Chapel Hill, Duke-NUS, and the Sanford-Burnham Medical Research Institute are handled not by members of the editorial board but rather by the science editors, who consult with selected external editors and reviewers.

Conflict of interest: The authors have declared that no conflict of interest exists. Submitted: September 6, 2013; Accepted: June 6, 2014.
}

Reference information: / Clin Invest. 2014;124(9):3741-3756. doi:10.1172/JCI73093. and HNC, AKT signaling is frequently activated through amplification and mutation of EGFR, mutation of PI3KCA, or loss of PTEN $(1,7)$. In prostate and breast cancers, AKT can be activated through ubiquitination by the IGF/TNF receptor-associated factor 6 (IGF/TRAF6) axis or the Her2/SKP2 axis, respectively $(8,9)$. TRAF6 is activated by various receptor-proximal protein interactions, which release its inherent autoinhibition (10) and indirectly activate PI3K via direct interaction with either Src or Ras (11). The interaction with Src family kinases was shown to result in direct phosphorylation of TRAF6 (12).

In addition to the abnormally activated EGFR/AKT signaling axis and other oncogenic pathways identified in GBM and HNC $(2,3)$, there could be additional genes that are involved or act in parallel to established oncogenic signaling pathways that promote tumorigenesis. Using digital karyotyping and fluorescent in situ hybridization analyses of GBM samples, we found that the discoidin, $\underline{\mathrm{CU}} \underline{\mathrm{B}}$, and $\underline{\mathrm{LCCL}}$ domain-containing protein 2 gene

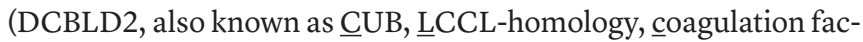
tor V/VIII homology domains protein 1 [CLCP1] and endothelial and smooth muscle cell-derived neuropilin-like protein [ESDN]) is amplified in several clinical GBM samples. DCBLD2 is a neuropilin-like membrane protein that was initially identified as an 
A

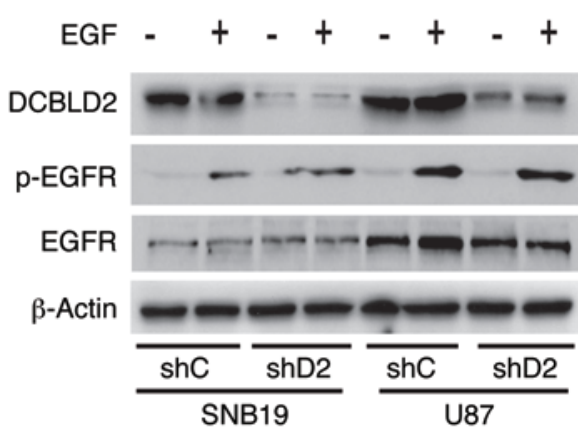

B

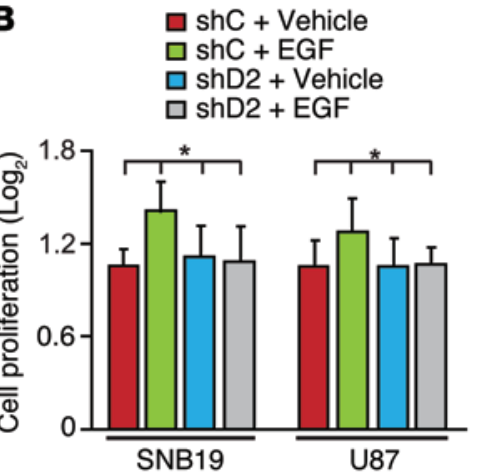
C $\quad \mathrm{shC}+$ Vehicle
$\square \mathrm{shC}+\mathrm{EGF}$
a shD2 + Vehicle
a shD2 + EGF

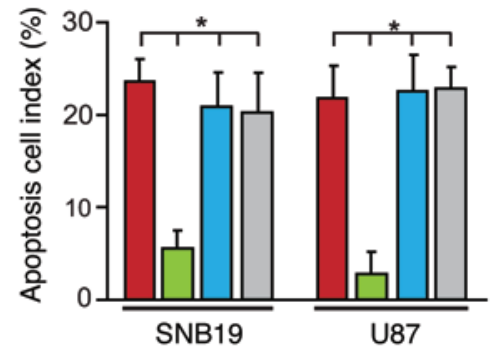

D

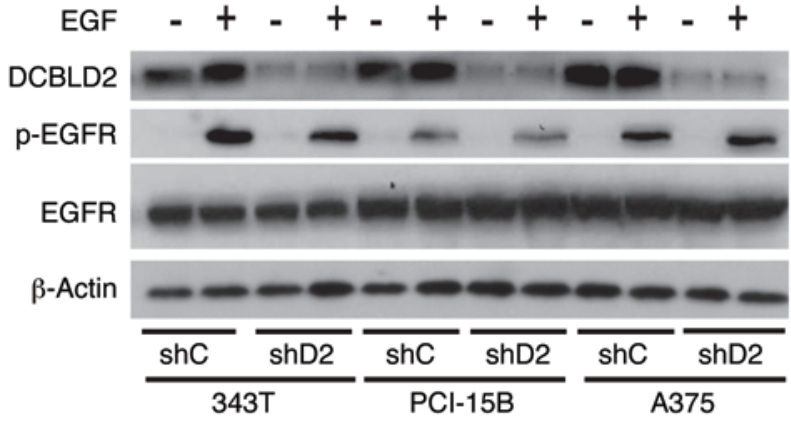

E

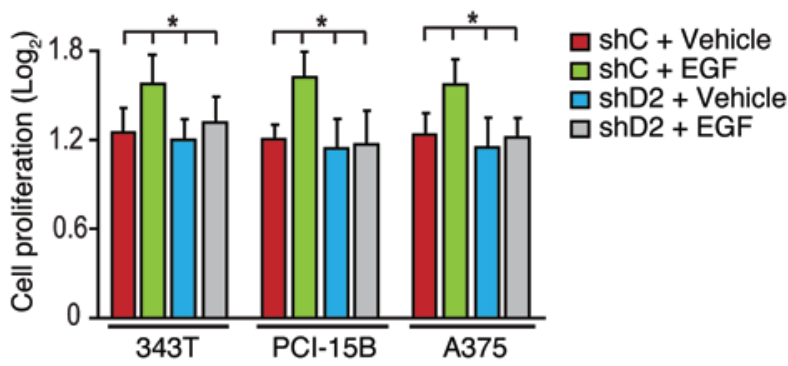

$\mathbf{F}$

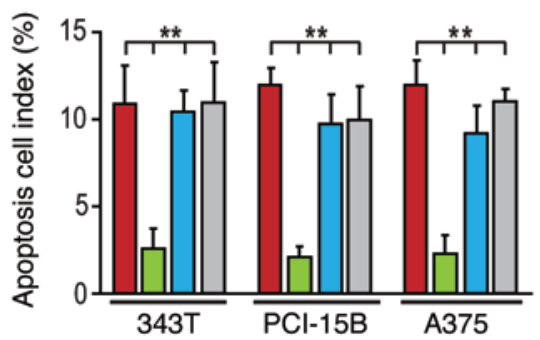

shC + Vehicle

$\square \mathrm{shC}+\mathrm{EGF}$

a shD2 + Vehicle

口 shD2 + EGF

G

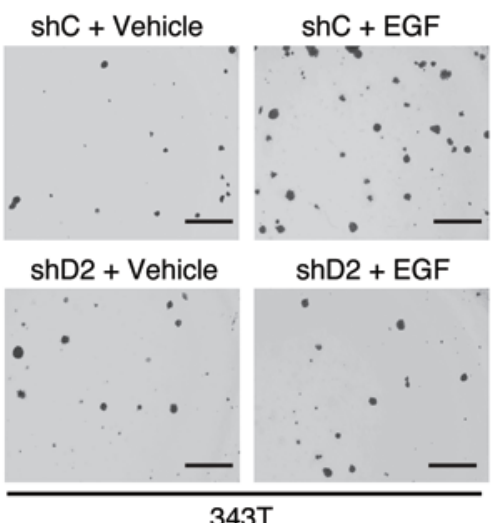

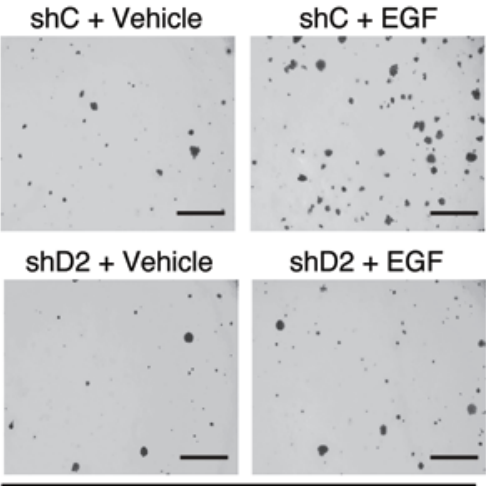

$\mathrm{PCl}-15 \mathrm{~B}$
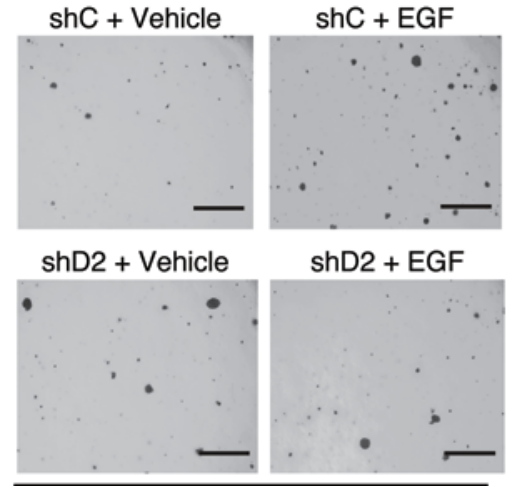

A375
H

$\square \mathrm{shC}+$ Vehicle $\square \mathrm{ShC}+\mathrm{EGF}$

$\square$ shD2 + Vehicle

$\square \mathrm{shD2}+\mathrm{EGF}$

语

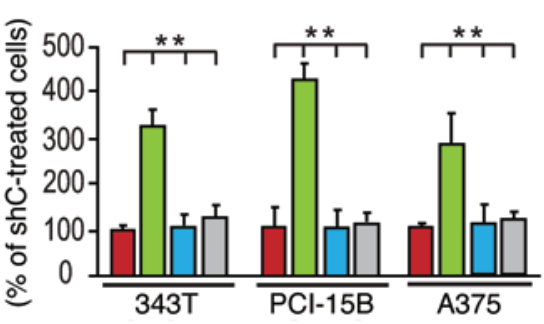

Figure 1. DCBLD2 is required for EGF-stimulated cell proliferation and survival in cancer cell lines derived from glioma, lung cancer, HNC, and melanoma. (A) DCBLD2 knockdown with a DCBLD2 shRNA (shD2) or a control shRNA (shC). EGF stimulation $(50 \mathrm{ng} / \mathrm{ml}$ ) of glioma SNB19 and U87 cells for 3 days. (B) Knockdown of DCBLD2 attenuates EGF-stimulated glioma cell proliferation. Clioma cells in 6 replicates were serum starved for 24 hours and then treated with or without EGF $(50 \mathrm{ng} / \mathrm{ml})$ for 3 days. (C) Knockdown of DCBLD2 inhibits glioma cell survival. (D) Knockdown of DCBLD2 by a shRNA (shD2) or a control shRNA (shC) in cell lines derived from lung cancer (343T), HNC (PCI-15B), and melanoma (A375). (E) Knockdown of DCBLD2 by shD2 inhibits EGF-stimulated 343T, PCI-15B, and A375 cell proliferation in vitro. (F) Effect of shRNA knockdown of DCBLD2 by shD2 on cell survival in vitro. (G) Effect of shRNA knockdown of DCBLD2 by shD2 on colony formation by $343 \mathrm{~T}, \mathrm{PCI}-15 \mathrm{~B}$, and $\mathrm{A} 375$ cells seeded on soft agar in triplicates. Scale bars: $1 \mathrm{~mm}$. (H) Quantification of colony formation assays in G. DCBLD2 was knocked down by 2 separate shRNAs (shD2\#1 and shD2\#2; see Figure 2) in all experiments and only results of shD2\#1 knockdown are shown. In B, C, E, F, and $\mathbf{H}$, error bars $\pm \mathrm{SD}$. ${ }^{*} P<0.05$, ${ }^{* *} P<0.01$, compared with parental with shC+EGF. Data and images are representative of 3 independent experiments. In $\mathbf{A}$ and $\mathbf{D}, \beta$-actin and EGFR were used as loading controls. 
upregulated protein in vascular injury (13). In vascular smooth muscle cells, DCBLD2 modulates PDGFR- $\beta$ stimulation by affecting ubiquitination of PDGFR- $\beta$ through c-CBL E3 ligase (14). In lung cancers, DCBLD2 is upregulated in LNM35 cells in association with its acquisition of a metastatic phenotype during in vivo selection, and it is also increased in a significant fraction of lung cancer samples, with a particularly high frequency in metastatic lesions (15). On the other hand, in clinical specimens of gastric and neuroendocrine cancers, DCBLD2 was found to be downregulated $(16,17)$, and ectopic expression of DCBLD2 in gastric cancer cell lines inhibited colony formation and cell invasion, suggesting a tumor suppressive role for DCBLD2 in these cancers. DCBLD2 is also linked to several human diseases (18). To date, cumulative evidence for the role of DCBLD2 in cancers and other human diseases is conflicting and limited. Moreover, proteomic studies of EGFR/EGFRvIII stimulation of various types of cancer cells have identified DCBLD2 as a phosphorylated protein at several tyrosine residues (19-21), suggesting a potential involvement of DCBLD2 in EGFR stimulation of cancer cell behavior.

In this study, we investigated the role of DCBLD2 in EGFR/ EGFRvIII-driven tumorigenesis. We found that DCBLD2 expression is increased in a large number of human GBMs. DCBLD2 is required for the EGFR-stimulated oncogenic behavior of cell lines derived from human gliomas, lung cancers, HNCs, and melanomas. EGFR phosphorylates tyrosine $(\mathrm{p}-\mathrm{Y})$ of the $\mathrm{Y} 750$ residue in DCBLD2. Moreover, $\mathrm{p}-\mathrm{Y} 750$ of DCBLD2 (p-DCBLD2 ${ }^{\mathrm{Y} 750}$ ) is located in a consensus TRAF6-binding motif (TIM) and mediates EGFR/EGFRvIII oncogenic signaling through interaction with TRAF6. This subsequently stimulates TRAF6 E3 ligase activity and activates AKT. The importance of this novel pathway is underlined by the coexpression of $\mathrm{p}-\mathrm{EGFR}^{\mathrm{Y} 1172}$, p-DCBLD2 ${ }^{\mathrm{Y} 750}$, TRAF6, and p-AKT ${ }^{\mathrm{T} 308}$ in a large number of glioma and HNC clinical samples. Coexpression of $\mathrm{p}-\mathrm{EGFR}^{\mathrm{Y} 1172}$ and $\mathrm{p}-\mathrm{DCBLD} 2^{\mathrm{Y} 750}$ also correlates with decreased survival of patients with gliomas or HNCs. Taken together, these results describe an important and novel signal relay by which EGFR/EGFRvIII phosphorylates p-DCBLD2 ${ }^{\mathrm{Y} 750}$, recruits TRAF6, and activates AKT oncogenic signaling, leading to enhanced tumorigenesis.

\section{Results}

Expression of DCBLD2 gene is upregulated in clinical GBMs. To identify potential oncogenic gene candidates in GBMs, we performed digital karyotyping analyses of 10 clinical GBM samples and found an amplification of 3q12.1 in GBM sample TB2580 (human chr3:99,801,814-100,181,106 Mb; University of California, Santa Cruz Genome Browser, v122). This amplified region contains the genes $D C B L D 2$ and ST3 $\beta$-galactosidase $\alpha-2,3$ sialyltransferase 6 (ST3GAL6). An adjacent second smaller peak did not include any known coding sequence (Supplemental Figure 1A; supplemental material available online with this article; doi:10.1172/ JCI73093DS1). To corroborate these observations, quantitative real-time reverse transcription PCR (Q-PCR) analyses of $28 \mathrm{GBM}$ samples were carried out to determine the generality of gene expression levels of DCBLD2 and ST3GAL6 in GBMs. As shown in Supplemental Figure 1B, DCBLD2, but not ST3GAL6, was expressed at high levels in 14 of the 28 GBMs (Supplemental Figure 1B). Serial analysis of gene expression (SAGE) further revealed an increased expression of DCBLD2 in gliomas of WHO tumor grade II-IV when compared with that in normal brain tissues (Supplemental Figure 1, C and D).

Recent genomic analyses suggest that GBM can be classified into 4 clinically relevant subtypes (proneural, neural, classical, and mesenchymal), and distinct signals are activated in these individual GBM subtypes that may account for the observed differential response to therapy (22). Thus, we examined gene expression and genomic data acquired from The Cancer Genome Atlas (TCGA) data portal across these GBM subtypes $(2,22)$. We found that expression of DCBLD2 was elevated in the majority of TCGA GBMs, including tumors from all GBM subtypes (Supplemental Figure 1E). High level DCBLD2 overexpression was most strongly associated with mesenchymal subtype tumors (Supplemental Figure $1 \mathrm{E}$ ), which have also been previously shown to highly express genes in the TNF super family pathway (22). Last, we performed in situ hybridization analysis of 3 clinical GBM samples, including TB2580, and found that DCBLD2 gene is amplified in these 3 clinical GBM cases (Supplemental Figure $1 \mathrm{~F}$ and data not shown). Taken together, these data show that expression of DCBLD2 is upregulated in GBMs and that amplification of DCBLD2 gene is detected in clinical human GBM tumors.

DCBLD2 is required for EGF-stimulated cell proliferation in cancer cell lines derived from glioma, lung cancer, HNC, and melanoma. The role of DCBLD2 in human cancers has not been fully elucidated (15-17) or studied in GBMs. To determine the roles of DCBLD2 in GBM and other types of human cancers, we examined protein expression of DCBLD2 in cancer cell lines derived from gliomas, HNCs, lung cancers, and melanomas. As shown in Supplemental Figure 2, DCBLD2 was expressed at high levels in 9 glioma cell lines, HNC line PCI-158, lung cancer lines 343T and $\mathrm{H} 3255$, and melanoma line A375. We then knocked down endogenous DCBLD2 in U87, SNB19, and LN444 glioma cells using 2 separate shRNAs and found that this had no effect on their in vitro proliferation and resulted in modest inhibition of in vivo growth of orthotopic U87 glioma xenografts (Supplemental Figure 3).

It is established that oncogenic EGFR/AKT signaling stimulates tumorigenesis of GBMs, HNCs, lung cancers, and melanomas $(3,4,6,23)$. Using a proteomics approach, we and others reported that EGF stimulation of EGFR or EGFRvIII, a constitutively active EGFR mutant that is frequently overexpressed in clinical GBMs and HNCs $(3,6,24)$, promotes phosphorylation of DCBLD2 at several tyrosine residues, including Y621 and Y750, in mammary epithelial cells, glioma, and lung cancer cell lines (19-21). Thus, we hypothesized that DCBLD2 might be involved in EGFR/AKT-promoted tumorigenesis in these cancers. Additionally, since TRAF6 activates AKT through its E3 ubiquitin ligase activity (8), we examined the expression levels of endogenous EGFR, DCBLD2, and TRAF6 in cell lines derived from these human cancers. We found that DCBLD2 is coexpressed with EGFR and TRAF6 in cancer cell lines derived from gliomas (U87, SNB19, LN229, T98G, D54, and LN444), HNCs (PCI-15B, Cal-33, and OSC-19), lung cancers (343T and H3255), and melanomas (A375) (Supplemental Figure 2).

Since amplification and mutation of EGFR are frequently found in clinical GBMs, lung cancers, and HNCS and aberrant EGF/AKT signaling drives tumorigenesis of GBMs, HNCs, lung 
A

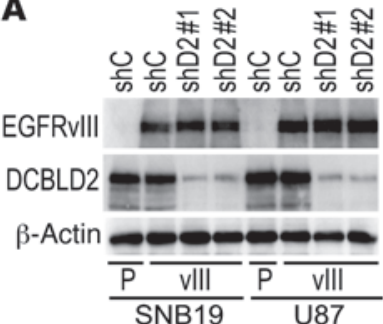

B

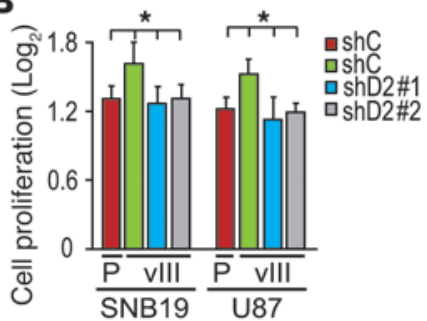

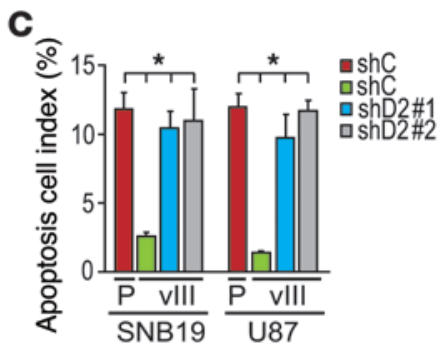

D

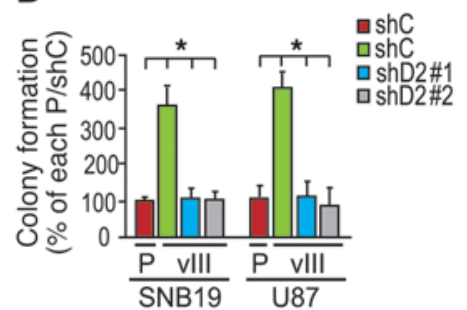

E
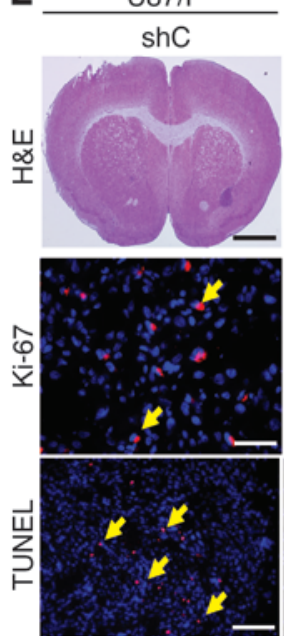
U87/vIII
shD2\#1

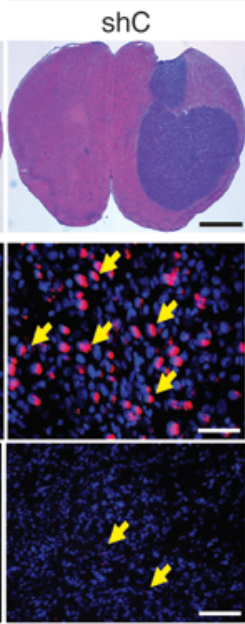

F

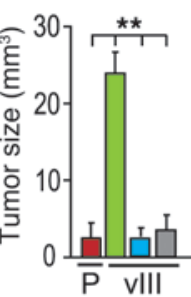

shD2\#2

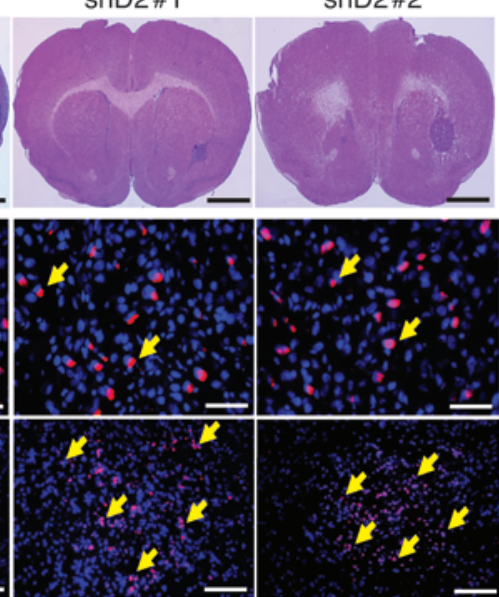

- P/shC

avlll/shC

avill/shD2\#1

口vlli/shD2\#2
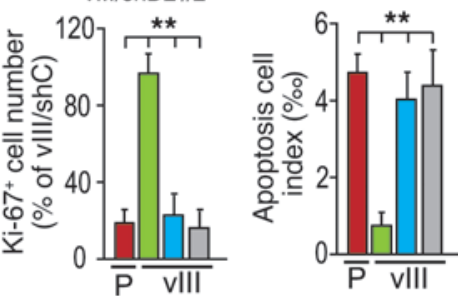

G

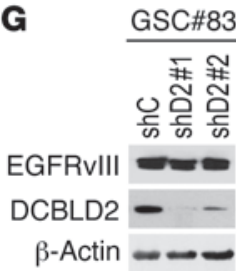

H

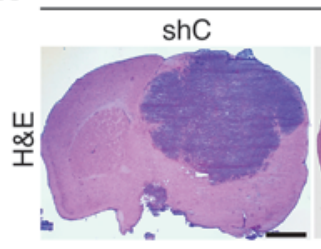

GSC\#83

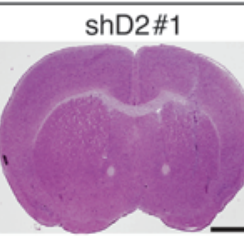

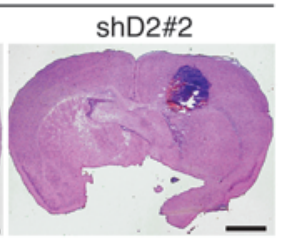

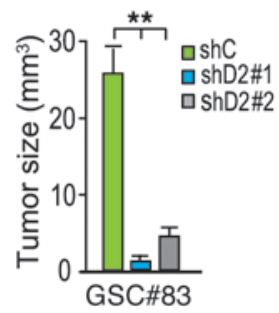

Figure 2. DCBLD2 is required for EGFR-driven tumorigenesis. (A) IB analyses of DCBLD2 knockdown with 2 different shRNAs (shD2\#1 and shD2\#2) or a control shRNA in U87 and SNB19 cells. P, parental cells; vIll, U87 or SNB19 cells expressing EGFRvIll. $\beta$-Actin was used as a loading control. (B) Effects of knockdown of DCBLD2 by shD2 or shC on cell proliferation in vitro. (C) Effects of knockdown of DCBLD2 by shD2 or shC on cell apoptosis in vitro. (D) Effect of DCBLD2 knockdown by shD2 or shC on glioma cell colony formation in vitro. (E) shRNA knockdown of DCBLD2 inhibits ECFRvlll-promoted U87 glioma growth in the brain. Representative images of H\&E, Ki-67, and TUNEL analyses of brain sections, with indicated U87 gliomas (arrows). Nuclei were stained with DAPI (blue). Ki-67 and TUNEL are in red. Scale bars: $1 \mathrm{~mm}$ (H\&E staining); $50 \mu \mathrm{m}$ (Ki-67 staining); $100 \mu \mathrm{m}$ (TUNEL staining). Images represent results of 5 mice per group. (F) Quantification of tumor size, cell proliferation, and cell apoptosis. Data were from stained brain sections of 5 mice per group. (C) IB analyses of DCBLD2 knockdown by shD2 or shC in patient-derived GSCs (GSC83). (H) shRNA knockdown of DCBLD2 inhibits endogenous ECFRvIIIpromoted tumorigenesis of gliomas established by patient-derived GSC83 cells in the brain. Quantification of tumor size is also shown. Scale bars: $1 \mathrm{~mm}$. In $\mathbf{B}, \mathbf{C}, \mathbf{D}, \mathbf{F}$, and $\mathbf{H}$, error bars $\pm \mathrm{SD} .{ }^{*} P<0.05$, ${ }^{* *} P<0.01$, compared with parental or EGFRvill cells or tumors treated with shC. Data and images are representative of 2 to 3 independent experiments.

cancers, and melanomas (3, 4, 6, 22, 23), we further hypothesized that DCBLD2 is critical for EGFR-driven tumorigenesis in human cancers associated with EGFR overexpression and activation. Toward this end, we knocked down endogenous DCBLD2 using shRNAs in SNB19 and U87 glioma cells (Figure 1A; see complete unedited blots in the supplemental material) and found that knockdown of DCBLD2 in these glioma cell lines inhibited EGF stimulation of cell proliferation, survival, and colony formation in vitro (Figure 1, B and C). Similarly, when endogenous DCBLD2 was depleted by shRNAs in 343T lung cancer, PCI-15B HNC, and A375 melanoma cell lines (Figure 1D), EGF-stimulated cell pro- liferation, cell survival, and anchorage-independent growth in soft agar in vitro were also markedly attenuated (Figure 1, E-H). Thus, these data suggest that DCBLD2 is involved in EGFR stimulation of cancer cell proliferation and survival.

We have demonstrated previously that the constitutively active, ligand-independent EGFRvIII promotes U87 and SNB19 tumorigenesis in the brains of animals $(25,26)$. To test whether DCBLD2 is critical for EGFRvIII-driven glioma tumorigenesis, we knocked down DCBLD2 in SNB19/EGFRvIII and U87/EGFRvIII cells using 2 separate shRNAs (Figure 2A). Depletion of DCBLD2 in EGFRvIII-expressing cells markedly inhibited EGFRvIII-pro- 

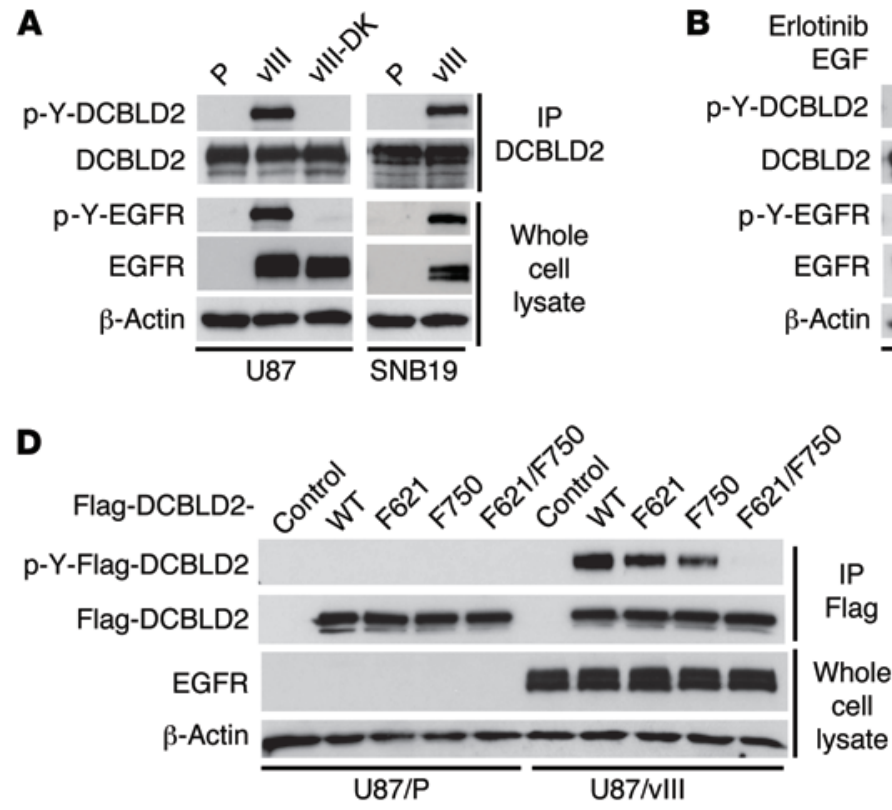

C

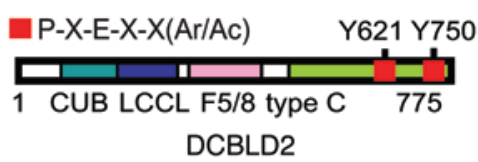

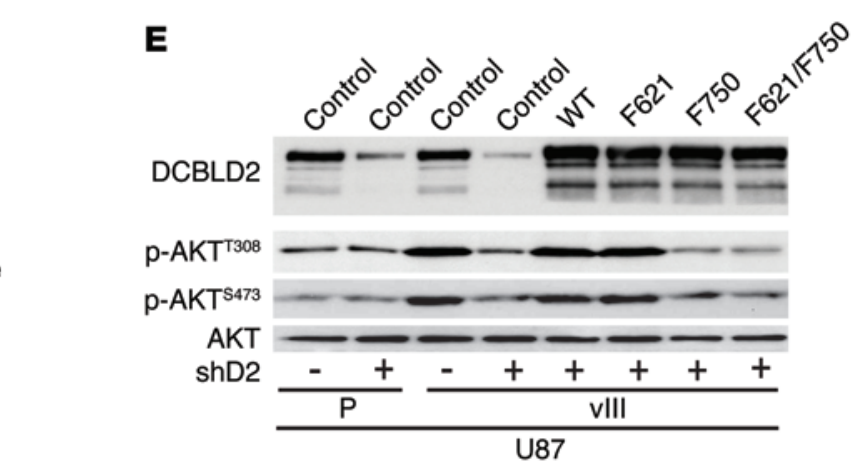

$\mathbf{F}$

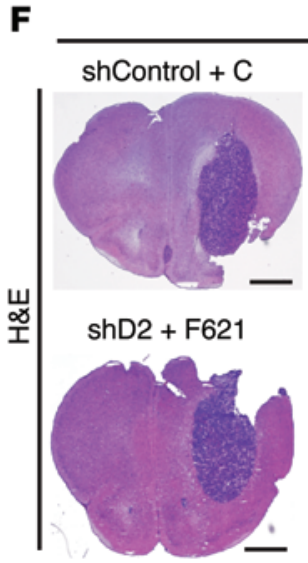

U87/VIII
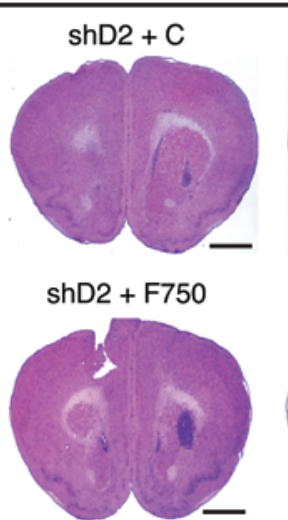

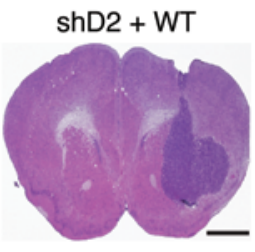

$\operatorname{shD} 2+\mathrm{F} 621 / \mathrm{F} 750$

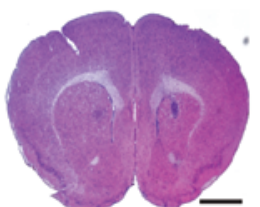

G

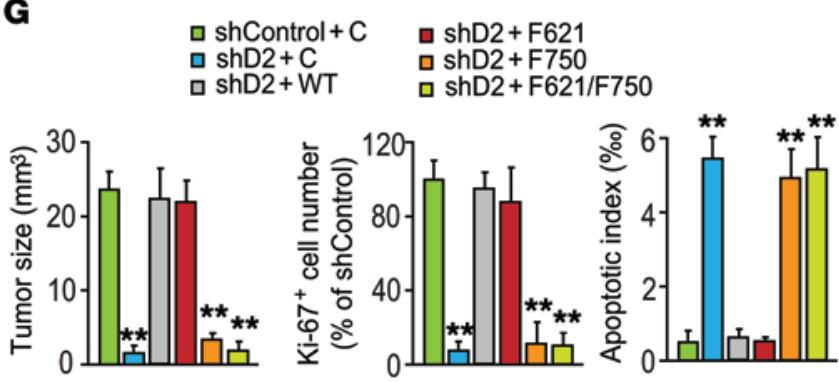

Figure 3. EGFR phosphorylation of DCBLD2 at Y750, but not at Y621, is critical for EGFR-promoted tumorigenesis. (A) ECFRvIII activates p-Y of DCBLD2 in glioma U87 and SNB19 cells. vIII or vIII-DK, U87 or SNB19 cells that express vlll or a mutant of vlll that lacks of cytoplasmic domain of vllI, respectively. $\mathrm{p}-\mathrm{Y}$ of DCBLD2 was detected with a pan anti-tyrosine antibody, 4G10. p-Y of EGFR was detected with an anti-p-EGFRY1045 antibody. (B) EGF (50 ng/ml) stimulates p-Y of DCBLD2 in U87/EGFR cells. (C) Schematic of the DCBLD2 ${ }^{\text {WT }}$ TIM P-X-E-X-X (Ar/Ac). CUB, C1r/C1s, Uegf, Bmp1 domain; LCCL, Limulus factor C, Coch-5b2, and Lgl1 domain; F5/8 type C, coagulation factor V/factor VIII homology (FV/VIII) domain. (D) DCBLD2 ${ }^{F 621 / F 750}$ mutant abolishes, and DCBLD2 ${ }^{F 621}$ or DCBLD2 ${ }^{\mathrm{F} 750}$ mutant attenuates, EGFRvill-stimulated p-Y of DCBLD2 in U87/P or U87/EGFRvill cells. (E) Reexpression of Flag-DCBLD2 shRNA-resistant DCBLD2 ${ }^{W T}$ or DCBLD2 ${ }^{F 621}$, but not DCBLD2 ${ }^{\mathrm{F} 750}$, DCBLD2 $2^{\mathrm{F} 621 / F 750}$ mutant or a vector control (C), rescues EGFRvill-stimulated $p-A K T^{T 308}$ and $p-A K T^{5473}$ in U87/ EGFRvIlI/shD2 cells. (F) Reexpression of Flag-DCBLD2 shRNA-resistant DCBLD2 ${ }^{\mathrm{WT}}$ and DCBLD2 ${ }^{\mathrm{F} 621}$, but not DCBLD2 ${ }^{\mathrm{F750}}$, DCBLD2 ${ }^{\mathrm{F} 621 / \mathrm{F} 750}$ mutant, or a vector control (C), rescues EGFRvIII-promoted U87 glioma growth in the brain of animals. Images represent results of 5 mice per group of 3 independent experiments. Scale bars: $1 \mathrm{~mm}$. (C) Effect of reexpression of Flag-DCBLD2 shRNA-resistant DCBLD2 ${ }^{\mathrm{WT}}$ or indicated mutants on tumor size, cell proliferation, and cell apoptosis in U87/EGFRvIll/shD2 tumors. Error bars \pm SD. ${ }^{* *} P<0.01$, compared with shControl $+C$ tumors or shD2 tumors expressing DCBLD2 ${ }^{W T}$ or DCBLD2 ${ }^{\text {F621 }}$ mutant. In A, B, D-G, data are representative of 3 independent experiments. $\beta$-Actin or AKT was used as a loading control.

moted cell proliferation, survival, and colony formation in soft agar in vitro (Figure 2, B-D, and Supplemental Figure 4A). When various engineered U87 cells were implanted into the brains of animals, knockdown of endogenous DCBLD2 by 2 separate shRNAs, but not a control shRNA, significantly reduced EGFRvIII-stimulated tumor growth, tumor cell proliferation, and cell survival (Figure 2, E and F). In patient-derived glioma stem cells (GSCs) (27, 28), we found that endogenous EGFRvIII is highly expressed in GSC line 83 (GSC83) and GSC1123 cells, whereas WT EGFR was detected in GSC528, JK18, and JK42 cells (Supplemental Figure 4, B and C). Since GSC83 and GSC1123 cells are highly tumorigenic in the brains of mice (27) and express DCBLD2 at high levels, we knocked down endogenous DCBLD2 using 2 separate shRNAs for DCBLD2 in both GSC lines. Inhibition of DCBLD2 markedly suppressed tumorigenesis in these intracranial xenografts, validating our observation in U87/EGFRvIII (Figure 2, G and H, and data not shown). Importantly, shRNA knockdown of endogenous DCBLD2 in various glioma, lung cancer, HNC, and melanoma cell lines had 


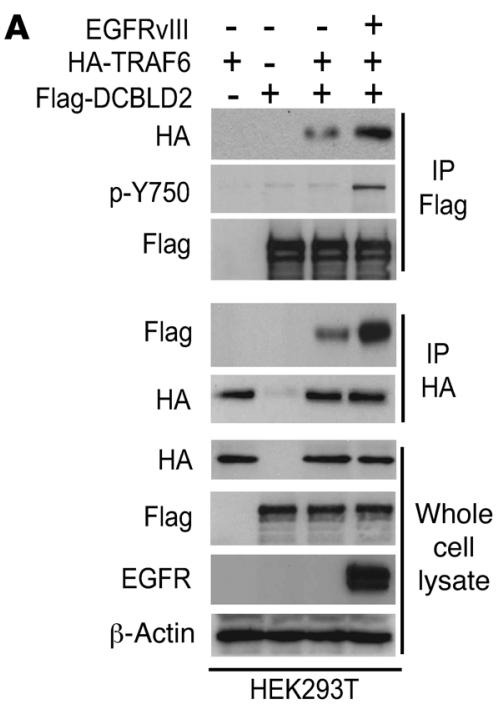

D

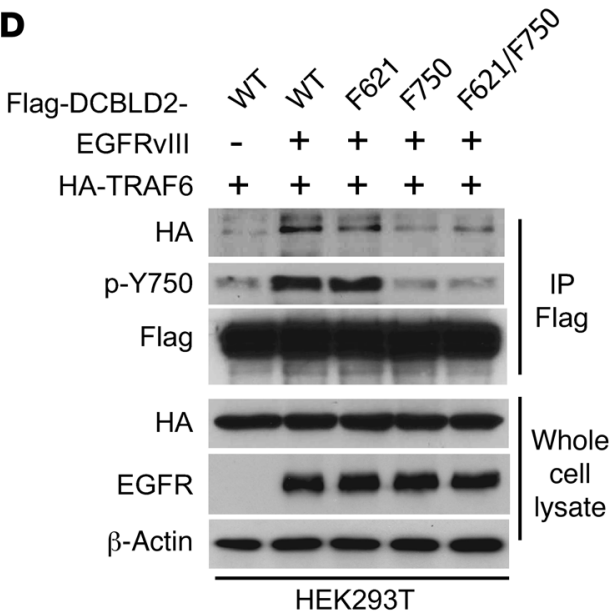

B

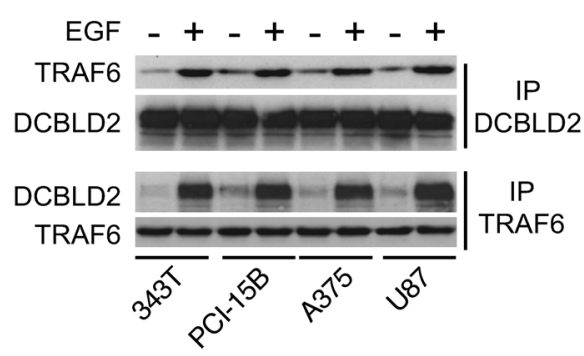

C

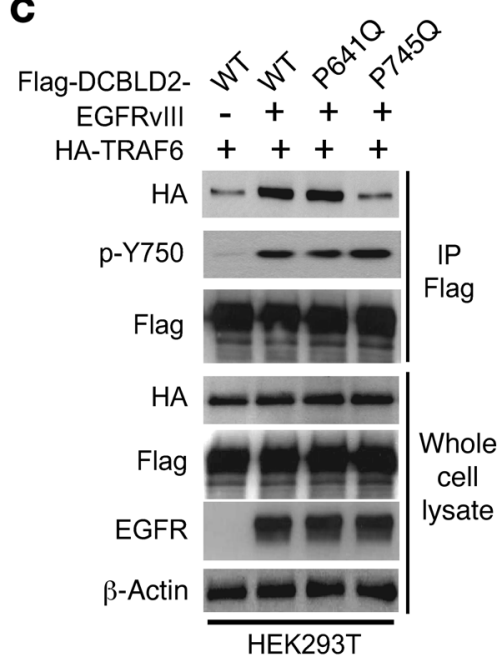

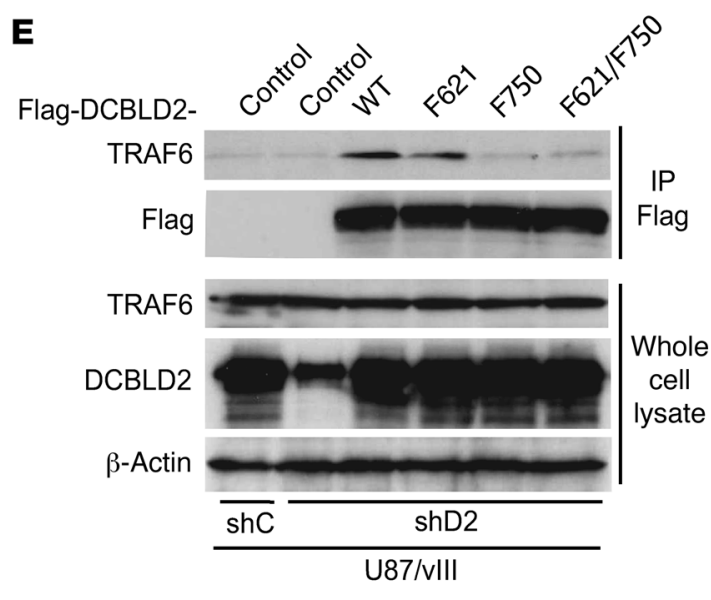

Figure 4. EGFR-stimulated p-DCBLD2 ${ }^{\mathrm{Y750}}$ regulates DCBLD2 association with TRAF6. (A) ECFRvIII p-DCBLD2 ${ }^{\mathrm{Y} 750}$ and promotes the association of DCBLD2 with TRAF6. HA-TRAF6 was coexpressed with or without Flag-DCBLD2 and/or EGFRvIII in HEK293T cells. (B) EGF stimulates DCBLD2 and TRAF6 association in lung cancer 343T, HNC PCI-15B, melanoma A375, and glioma U87 cells. (C) Mutation of P745Q, but not P641Q, of DCBLD2 TIMs impairs EGFRvIIIinduced association of DCBLD2 with TRAF6. (D) EGFR-stimulated p-DCBLD2 ${ }^{\mathrm{Y} 750}$ is critical for DCBLD2 binding to TRAF6. (E) EGFRvIII-activated p-Y750, but not p-Y621, of DCBLD2 is required for DCBLD2 binding to TRAF6. IP-IB or IB analyses. A specific anti-p-DCBLD2 ${ }^{\mathrm{Y750}}$ antibody was used to detect EGFR-stimulated p-DCBLD2 ${ }^{\mathrm{Y} 750}$. Control, vector without DCBLD2. $\beta$-Actin was used as a loading control. Data are representative of 3 independent experiments.

no effect on the expression of EGFR, AKT, or $\beta$-actin, thus excluding off-target effects of the shRNA constructs (Figure 1, A and D, and Figure 2, A and G). Collectively, these findings suggest that DCBLD2 plays an important role in EGFR/EGFRvIII-driven tumorigenesis in human cancers.

EGFR phosphorylation of DCBLD2 at Y750, but not Y621, is critical for EGFR-driven tumorigenesis. Overexpressed or mutated EGFR drives tumorigenesis and progression of various types of human cancers through activation of AKT signaling $(2,5,6,23)$. Since EGF or constitutively activated EGFRvIII phosphorylates DCBLD2 at Y621 and Y750 in glioma and lung cancer cells (20, 21), we hypothesized that EGFR- and EGFRvIII-dependent $\mathrm{p}-\mathrm{Y}$ of DCBLD2 is critical for EGFR-driven tumorigenesis in human cancers associated with EGFR activation. To test this, we used a pan anti-p-Y antibody, 4G10, and examined p-Y of DCBLD2. As shown in Figure 3, A and B, and Supplemental Figure 5, expression of EGFRvIII in U87 and SNB19 glioma cell lines or EGF stimula- tion of U87 glioma cells, 343T lung cancer cells, PCI-15B HNC, and A375 melanoma cells, promoted p-Y of DCBLD2. Moreover, treatment with the EGFR tyrosine kinase inhibitor, erlotinib, suppressed $\mathrm{p}-\mathrm{Y}$ of DCBLD2 in vitro. However, we were unable to detect a direct association between DCBLD2 and EGFR in U87/ EGFR WT cells by reciprocal immunoprecipitation and immunoblotting (IP-IB) analyses (Supplemental Figure 6).

Since DCBLD2 does not contain any signaling module in its cytoplasmic domain (13), we performed in silico analysis through The Eukaryotic Linear Motif Resource for Functional Sites of Proteins (http://elm.eu.org) and identified 2 potential consensus TIMs in DCBLD2, FKPEEGKEA and PAPDELVYQ (bold font represents consensus amino acid [AA] residues in these sequences) (29), at AA residues 639-647 and 743-751, respectively (Figure 3C). Significantly, these 2 conserved sequences are next to, or encompass, the EGFR-dependent p-Y621 and p-Y750 residues of DCBLD2, suggesting a potential interaction of tyrosine-phosphorylated 
A

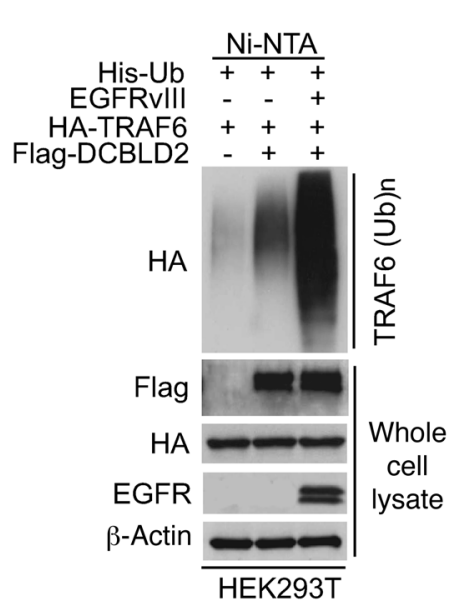

C

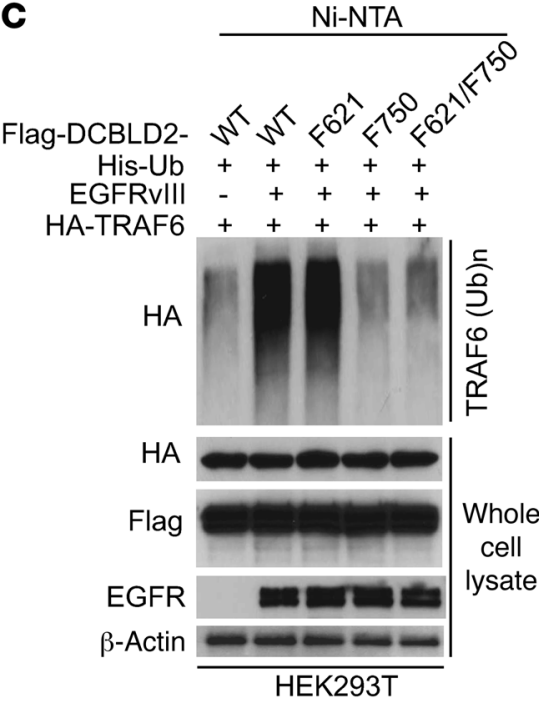

B

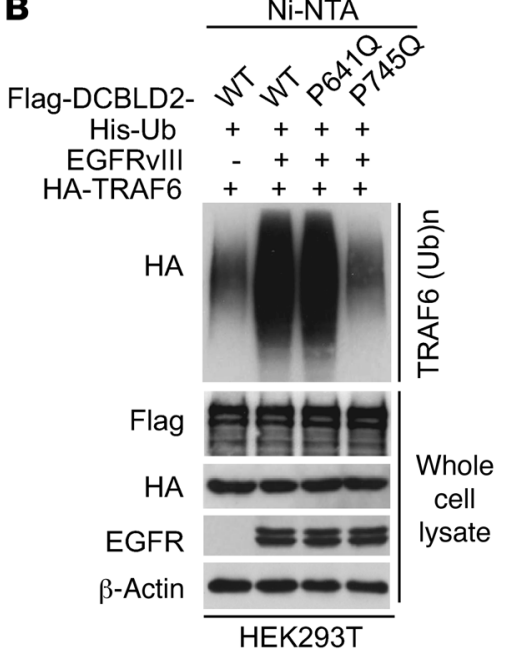

D

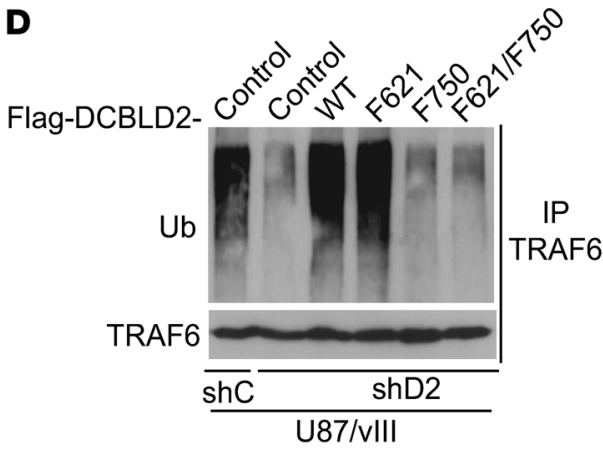

Figure 5. EGFR-stimulated p-DCBLD2 ${ }^{7750}$ regulates TRAF6 E3 ligase activity. IP-IB or IB analyses. (A) DCBLD2 promotes EGFR-stimulated TRAF6 E3 ligase activity. HA-TRAF6 and His-Ub were coexpressed with or without Flag-DCBLD2 and/or EGFRvIll in HEK293T cells. Proteins were pulled down with Ni-NTA beads. TRAF6 (Ub)n, polyubiquitinated TRAF6. (B) Mutation of P745Q, but not P641Q, in DCBLD2 inhibits EGFRvIll-stimulated TRAF6 E3 ligase activity. (C) Compared with DCBLD2 ${ }^{\mathrm{WT}}$, expression of DCBLD2 $2^{\mathrm{F} 750}$ or DCBLD2 $2^{\mathrm{F} 621 / \mathrm{F} 750}$, but not DCBLD2 ${ }^{F 621}$, attenuates EGFRvIII-stimulated TRAF6 E3 ligase activity. (D) Reexpression of DCBLD2 ${ }^{\mathrm{WT}}$ or DCBLD2 ${ }^{\mathrm{F} 621}$, but not DCBLD2 ${ }^{\mathrm{F} 750}$ or DCBLD2 $2^{\mathrm{F} 621 / \mathrm{F} 750}$, rescues EGFRvIll-stimulated TRAF6 E3 ligase activity in glioma U87/ EGFRvIII/shD2 cells. HA-TRAF6, Flag-DCBLD2, EGFR, and $\beta$-actin were used as loading controls. Data are representative of 3 independent experiments with similar results.
DBCLD2 with TRAF6. Therefore, we evaluated whether EGFR/ EGFRvIII-dependent $\mathrm{p}-\mathrm{Y}$ of Y621 and Y750 of DCBLD2 is critical in EGFR-promoted tumorigenesis. We coexpressed in U87 parental and U87/EGFRvIII cells DCBLD2 WT (DCBLD2 ${ }^{\mathrm{WT}}$ ) along with one of its mutant forms, DCBLD2 $2^{\mathrm{F} 621}$, DCBLD2 $2^{\mathrm{F} 750}$, or DCBLD2 $2^{\mathrm{F} 621 / \mathrm{F} 750}$, in which $\mathrm{Y}$ residue(s) was changed to a nonphosphorylatable phenylalanine (F) residue(s). Double mutation of Y621F/Y750F abolished EGFR-dependent $\mathrm{p}-\mathrm{Y}$, while individual mutation of $\mathrm{Y} 621 \mathrm{~F}$ or Y750F reduced $\mathrm{p}-\mathrm{Y}$ of DCBLD2 (Figure 3D). Next, we separately expressed shRNA-resistant DCBLD2 ${ }^{\mathrm{WT}}, \mathrm{DCBLD} 2^{\mathrm{F} 621}, \mathrm{DCBLD}^{\mathrm{F} 750}$, or DCBLD2 ${ }^{\mathrm{F} 621 / \mathrm{F} 750}$ mutants in U87/EGFRvIII/shD2 cells, in which endogenous DCBLD2 was stably knocked down. Reexpression of DCBLD2 ${ }^{\mathrm{WT}}$ or the DCBLD2 ${ }^{\mathrm{F} 621}$ mutant rescued EGFRvIII-dependent $\mathrm{p}-\mathrm{AKT}^{\mathrm{T} 308}$ and $\mathrm{p}-\mathrm{AKT}^{\mathrm{S} 473}$, as well as colony formation in soft agar in vitro, but had no effect on p-ERK1/2 levels. In contrast, the DCBLD2 $2^{\mathrm{F} 750}$ or DCBLD2 $2^{\mathrm{F} 621 / \mathrm{F} 750}$ mutants failed to rescue EGFRvIII-promoted oncogenic signaling and tumorigenic behavior of glioma cells in vitro (Figure $3 \mathrm{E}$ and Supplemental Figures 7 and 8). When these engineered U87/EGFRvIII cells were implanted into the brains of animals, DCBLD2 ${ }^{\mathrm{WT}}$ or the DCBLD2 ${ }^{\mathrm{F} 621}$ mutant, but not the $\mathrm{DCBLD} 2^{\mathrm{F} 750}$ or $\mathrm{DCBLD} 2^{\mathrm{F} 621 / \mathrm{F} 750}$ mutants, rescued
EGFRvIII-enhanced U87 tumorigenesis in the brain (Figure 3, $\mathrm{F}$ and $\mathrm{G}$ ). These results suggest that EGFR-dependent $\mathrm{p}-\mathrm{Y} 750$, but not $\mathrm{p}-\mathrm{Y} 621$, of DCBLD2 is required for EGFRvIII-stimulated oncogenic signaling and glioma tumorigenesis.

EGFR-dependent $p$-DCBLD2 $2^{Y 750}$ regulates $D C B L D 2$ association with TRAF6. Our in silico analysis also revealed that Y750, but not Y621, is included within the consensus TIM in DCBLD2, PAPDELVYQ (AA 743-751; "Y" in bold font represents Y750) (Figure 3C). We reported previously that an E3 ubiquitin ligase, TRAF6, regulates oncogenic AKT activities (8). We thus hypothesized that p-DCBLD2 ${ }^{\mathrm{Y} 750}$ mediates EGFR stimulation of AKT through interaction with TRAF6. To test this hypothesis, we generated a specific anti-p-DCBLD2 ${ }^{\mathrm{Y} 750}$ antibody and found that it detected EGFRvIII-dependent p-DCBLD2 ${ }^{\mathrm{Y} 750}$ in U87/EGFRvIII cells and in U87/EGFRvIII/shD2 cells expressing shRNA-resistant DCBLD $2^{\mathrm{WT}}$ and DCBLD2 $2^{\mathrm{F} 621}$ but not those expressing DCBLD2 $2^{\mathrm{F} 750}$ and DCBLD2 $2^{\mathrm{F} 621 / \mathrm{F} 750}$ (Supplemental Figure 9, A and B). The specificity of the antibody recognition of $\mathrm{p}-\mathrm{DCBLD} 2{ }^{\mathrm{Y} 750}$ was further validated by immunohistochemical (IHC) analyses of a clinical GBM specimen (Supplemental Figure 9C). Additionally, in vitro kinase assays using recombinant and active EGFR kinases showed that 
A
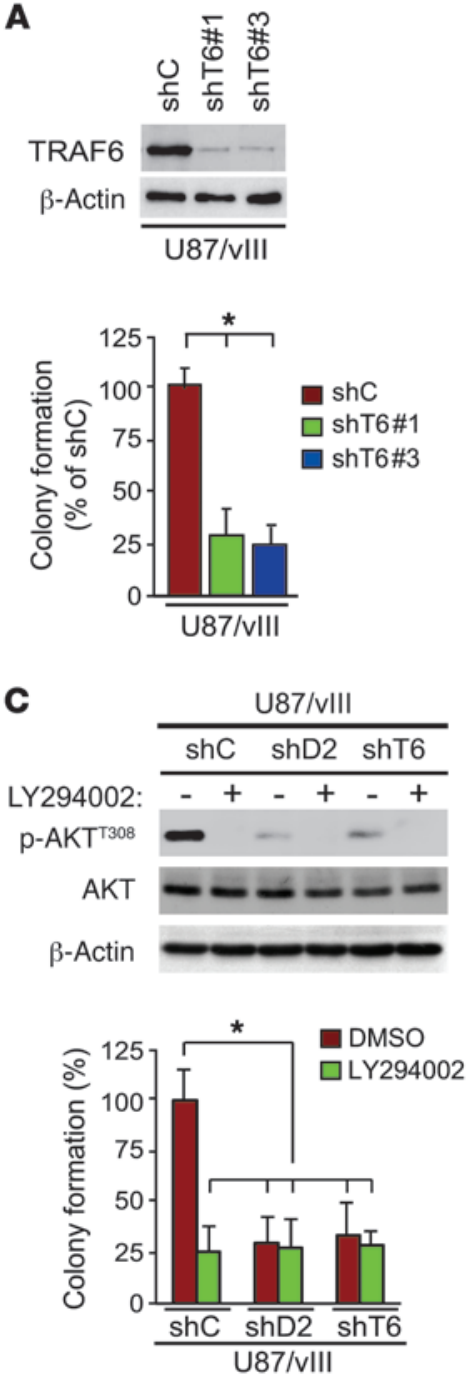

B
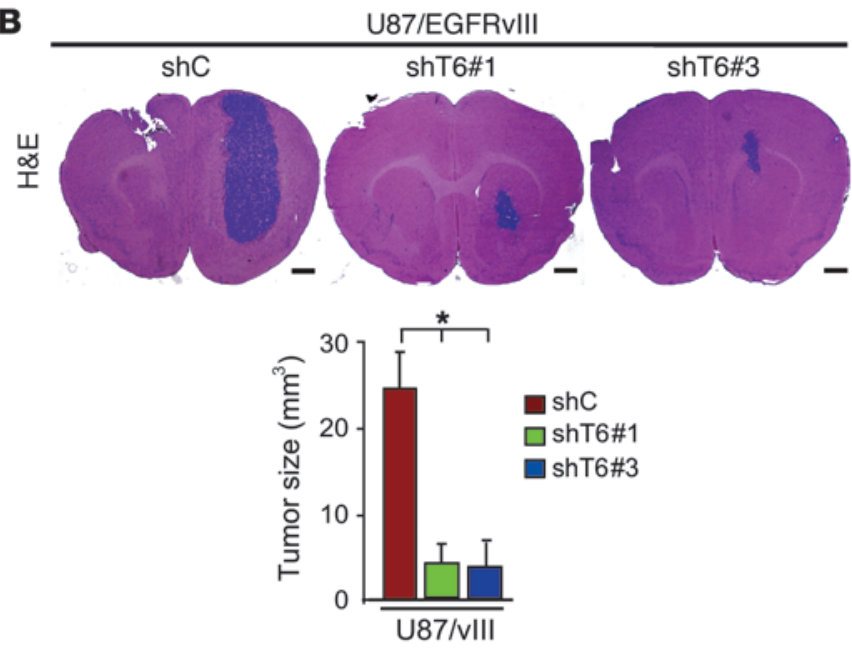

D
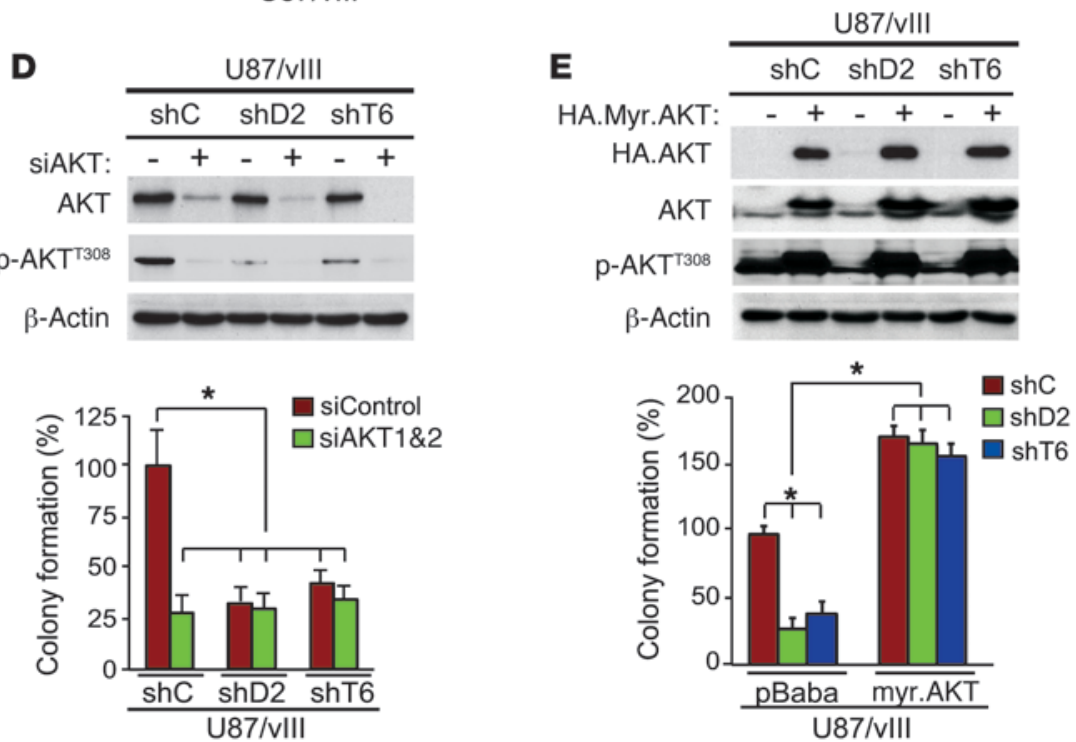

Figure 6. EGFRvIII/DCBLD2/TRAF6/AKT signal pathway regulates glioma tumorigenesis. (A) IB assays of knockdown of TRAF6 in U87/EGFRvIII cells with 2 different shRNAs, shT6\#1 and shT6\#3, and EGFRvlll-stimulated colony formation was inhibited by knockdown of TRAF6 with shT6. (B) Depletion of TRAF6 by shRNA inhibited EGFRvIII-promoted glioma tumorigenesis in the brains of animals. Scale bars: 1 mm. Images are representative of 5 mice per group of 2 independent experiments. (C) Treatment of U87/vIII cells with LY294002 inhibited ECFRvIII-stimulated AKT and colony formation, similar to knockdown of DCBLD2 (shD2) and TRAF6 (shT6) in U87/vllI cells. (D) Knockdown of AKT1 and AKT2 with siRNA (siAKT1\&2) in U87/vIII cells blocked EGFRvIll-stimulated activity of AKT and colony formation, similar to knockdown of DCBLD2 (shD2) and TRAF6 (shT6) in U87/vIll cells. (E) Overexpression of constitutively active form of AKT (Myr.AKT) not only further stimulated colony formation of U87/vIII/shC cells, but also rescued the inhibited colony formation by knockdown of DCBLD2 (shD2) or by knockdown of TRAF6 (shT6). $\beta$-Actin was used as a loading control. Cells were seeded in 6 replicates. Error bars $\pm \mathrm{SD}$. ${ }^{*} P<0.05$, compared with shC-treated cells (bars of shD2 and shT6 among pBABE) and compared with shC pBABE (bars of all myr. AKT). Data are representative of 2 independent experiments.

this antibody detected the activated EGFR-dependent p-Y750 in DCBLD2 ${ }^{\mathrm{WT}}$ but not the DCBLD2 ${ }^{\mathrm{F} 750}$ mutant (Supplemental Figure 10). These data indicate that the anti-p-DCBLD2 ${ }^{\mathrm{Y} 750}$ antibody is specific for $\mathrm{p}-\mathrm{DCBLD} 2^{\mathrm{Y} 750}$ in cells and tumor specimens and that activated EGFR directly phosphorylates DCBLD2 at Y750.

Next, we determined whether EGFR stimulates the interaction of DCBLD2 with TRAF6 through p-DCBLD2 ${ }^{\mathrm{Y} 750}$. As shown in Figure 4A, in human embryonic kidney 293T (HEK293T) cells without EGFRvIII expression, a modest association of DCBLD2 with TRAF6 was detected. In contrast, EGFRvIII expression in these cells markedly enhanced such an interaction and was accompanied by generation of $\mathrm{p}-\mathrm{DCBLD} 2^{\mathrm{Y} 750}$. In cancer cell lines derived from glioma (U87 and SNB19), lung cancer (343T), HNC
(PCI-15B), and melanoma (A375), EGF (but not HGF or PDGF-A) stimulation resulted in DCBLD2 interaction with TRAF6 and p-DCBLD2 $2^{\mathrm{Y} 750}$ (Figure $4 \mathrm{~B}$ and Supplemental Figure 11). To characterize the interaction of DCBLD2 with TRAF6, we generated separate mutations (P641Q and P745Q) within the 2 TIMs of DCBLD2 (Figure 3C) that match consensus motifs critical for TRAF6 binding in other proteins (29). Compared with DCBLD2 ${ }^{\mathrm{WT}}$, DCBLD2 $2^{\mathrm{P} 74 \mathrm{Q}}$, but not the DCBLD2 ${ }^{\mathrm{P} 641 \mathrm{Q}}$ mutant, displayed an appreciable decrease in association with TRAF6 (Figure 4C). Then, we examined the effects of $\mathrm{p}-\mathrm{Y}$ of DCBLD2 on EGFRvIIIstimulated DCBLD2 association with TRAF6. Compared with DCBLD2 ${ }^{\mathrm{WT}}, \mathrm{DCBLD} 2^{\mathrm{F} 750}$ and DCBLD2 ${ }^{\mathrm{F} 621 / \mathrm{F} 750}$ mutants showed markedly attenuated EGF-dependent associations, whereas 
A

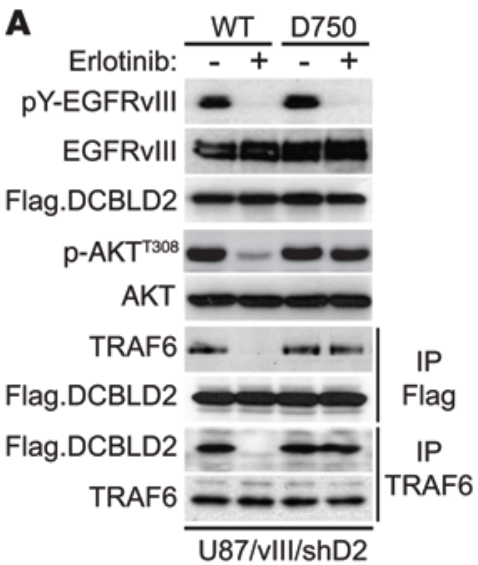

B

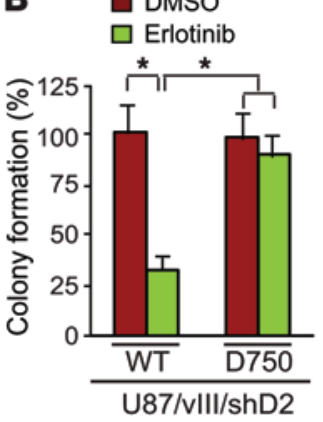

C

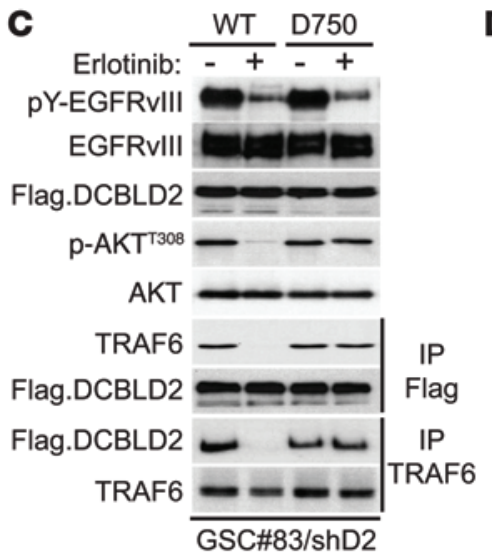

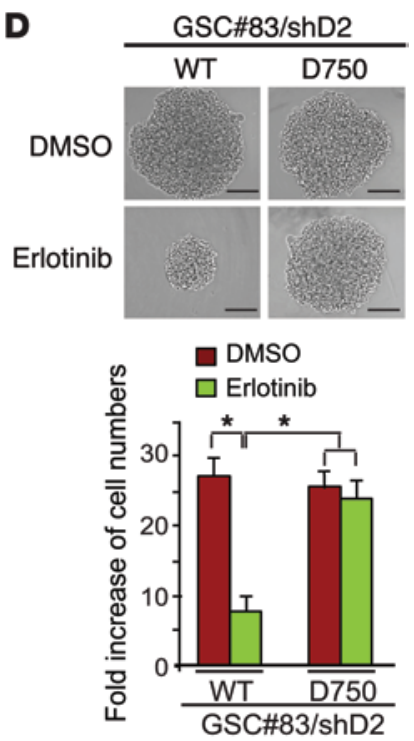

Figure 7. Mutation of DCBLD2 from Y750 to D750 mimics EGFRvIII signaling on AKT activation and tumorigenesis. (A) IP and/or IB assays of erlotinib treatments of U87/EGFRvIII/shD2 cells or (C) patient-derived GSC83/shD2 cells with exogenous expression of shRNA-resistant DCBLD2 ${ }^{\mathrm{WT}}$ or dominant activating DCBLD2 ${ }^{\mathrm{D} 750}$ mutant. (B) Erlotinib inhibited colony formation of U87/EGFRvIll/shD2/WT cells but had minimal effect on U87/EGFRvIII/shD2/ D750 cells. (D) Erlotinib attenuated size of neurosphere and fold increase of cell numbers of CSC83/shD2/WT cells after 5 days of cell culture but had no appreciable effect on GSC83/shD2/D750 cells. Cells were seeded in 6 replicates. Scale bars: $50 \mu \mathrm{m}$. In $\mathbf{B}$ and $\mathbf{D}$, error bars \pm SD. ${ }^{*} P<0.05$, compared with erlotinib-treated cells. In A and C, Flag-DCBLD2, EGFR and AKT were used as loading controls. Data are representative of 2 independent experiments.

DCBLD2 ${ }^{\mathrm{F} 621}$ had a minimal decrease in DCBLD2 association with TRAF6 (Figure 4D). Furthermore, reexpression of DCBLD2 ${ }^{\mathrm{WT}}$ and $\mathrm{DCBLD} 2^{\mathrm{F} 621}$, but not DCBLD2 $2^{\mathrm{F} 750}$ and $\mathrm{DCBLD} 2^{\mathrm{F} 621 / \mathrm{F} 750}$, rescued EGFRvIII-dependent binding of DCBLD2 to TRAF6 in glioma U87/EGFRvIII/shD2 cells (Figure 4E). DCBLD2 was also associated with TRAF6 in short-term primary cultures of GBM6 and GBM39 cells that have endogenous EGFRvIII overexpression. However, DCBLD2 is not associated with TRAF6 in GBM14 cells that have undetectable EGFR or EGFRvIII proteins $(30,31)$. Knockdown of endogenous DCBLD2 by shRNAs inhibited cell proliferation and colony formation of GBM6 and GBM39 cells (Supplemental Figure 12). Taken together, these findings suggest that the consensus PAPDELVYQ (AA 743-751; bold font indicates conserved amino residues in this sequence) motif of DCBLD2 is a major binding motif for TRAF6 and that EGFR stimulation of p-DCBLD $2{ }^{\mathrm{Y} 750}$ modulates their association.

EGFR-dependent $p$-DCBLD2 ${ }^{Y 750}$ regulates TRAF6 E3 ligase activity. We have shown previously that the E3 ligase, TRAF6, regulates AKT ubiquitination and activation, thereby promoting tumorigenesis (8). Thus, we tested the hypothesis that EGFR promotes tumorigenesis through phosphorylation of DCBLD2 ${ }^{\mathrm{Y} 750}$ that recruits TRAF6, ultimately leading to AKT activation. We found that EGFRvIII activated TRAF6 E3 ligase (Supplemental Figure 13). Loss of TRAF6 E3 ligase activity by a C70A mutation (29) did not affect EGFRvIII-dependent DCBLD2 binding to TRAF6 (Supplemental Figure 14). Moreover, DCBLD2 promoted TRAF6 E3 ligase activity when compared with control, and expression of EGFRvIII further enhanced this activity, suggesting that EGFRvIII-stimulated DCBLD2 interaction with TRAF6 regulates TRAF6 E3 ligase activity (Figure 5A). To solidify this conclusion, we coexpressed $\mathrm{DCBLD} 2^{\mathrm{WT}}, \mathrm{DCBLD} 2^{\mathrm{P} 641 \mathrm{Q}}$, or $\mathrm{DCBLD} 2^{\mathrm{P} 745 \mathrm{Q}}$ with
TRAF6, EGFRvIII, and His-Ub in HEK293T cells. Compared with DCBLD2 $2^{\mathrm{WT}}, \mathrm{DCBLD} 2^{\mathrm{P} 745 \mathrm{Q}}$, but not DCBLD2 $2^{\mathrm{P} 641 \mathrm{Q}}$, inhibited EGFRvIII-stimulated TRAF6 E3 ligase activity (Figure 5B). Coexpression of EGFRvIII with either DCBLD2 $2^{\mathrm{WT}}$ or DCBLD2 ${ }^{\mathrm{F} 621}$, but not with DCBLD2 $2^{\mathrm{F} 750}$ or DCBLD2 $2^{\mathrm{F} 621 / \mathrm{F} 750}$, stimulated TRAF6 E3 ligase activity (Figure $5 \mathrm{C}$ ), suggesting that $\mathrm{p}-\mathrm{DCBLD} 2^{\mathrm{Y} 750}$ is critical for EGFR/DCBLD2-activated TRAF6 E3 ligase activity. This observation was further underscored by separate reexpression of shRNA-resistant DCBLD2 ${ }^{\mathrm{WT}}$, DCBLD2 ${ }^{\mathrm{F} 621}$, DCBLD2 $2^{\mathrm{F} 750}$, or DCBLD2 $2^{\mathrm{F} 621 / \mathrm{F} 750}$ in U87/EGFRvIII/shD2 cells (Figure 5D). Therefore, these data suggest that EGFRvIII-dependent p-DCBLD2 ${ }^{\mathrm{Y} 750}$ regulates TRAF6 E3 ligase activity.

$T R A F 6$ and $p-D C B L D 2^{Y 750}$ are required for EGFRvIII/AKT-driven glioma tumorigenesis. TRAF6 was found to be an amplified oncogene in lung cancer and is important in Ras-mediated oncogenesis (32) and $\mathrm{PC}-3$ prostate cancer tumorigenesis (8). TRAF6 regulates in vitro cell proliferation, apoptosis, and invasion in cancer cell lines derived from gliomas and lung cancers $(33,34)$. Since our results show that TRAF6 mediates EGFR/EGFRvIII/DCBLD2-stimulated glioma tumorigenesis, we determined whether TRAF6 is required for EGFRvIII-promoted glioma tumorigenesis. We knocked down endogenous TRAF6 in U87/EGFRvIII cells using 2 separate shRNAs (Figure 6A). Depletion of TRAF6 by these shRNAs inhibited U87/EGFRvIII cell proliferation in vitro and markedly suppressed EGFRvIII-promoted tumorigenesis of glioma xenografts in the brains of animals (Figure 6B). These results indicate that TRAF6 is critical for EGFRvIII-driven glioma tumorigenesis.

We reported recently that TRAF6 regulates IGF-1-stimulated AKT oncogenic activity and promotes prostate cancer cell tumorigenesis (8) but TRAF6 is not involved in Her2-driven breast cancer tumorigenesis (9). Nonetheless, our data here demonstrate that TRAF6 
A

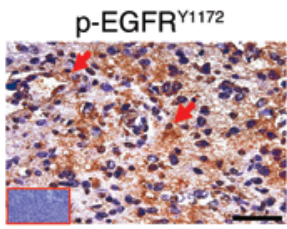

TRAF6

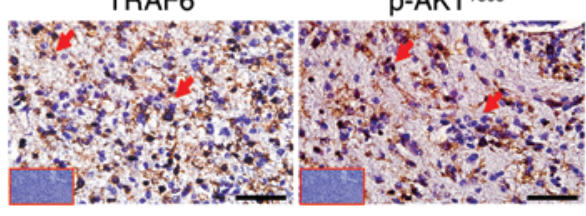

C

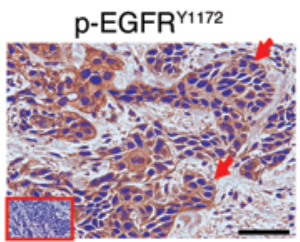

TRAF6

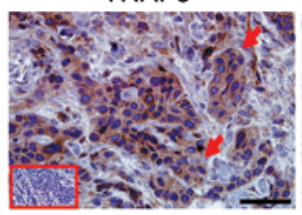

E

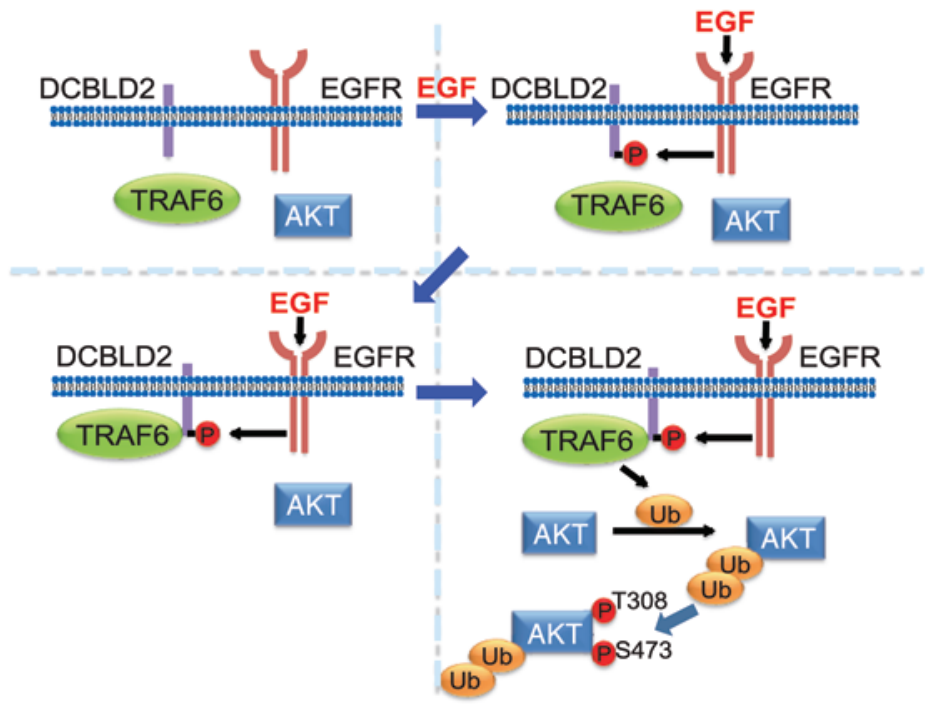

B

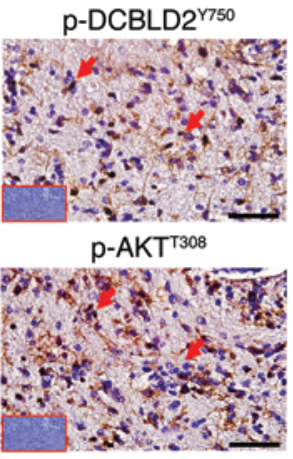

p-DCBLD2 $2^{\text {Y750 }}$

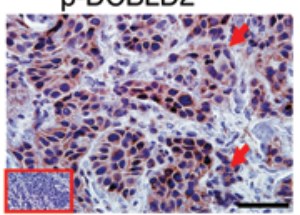
$\mathrm{p}-\mathrm{AKT}^{\mathrm{T} 308}$

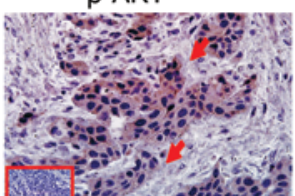

D
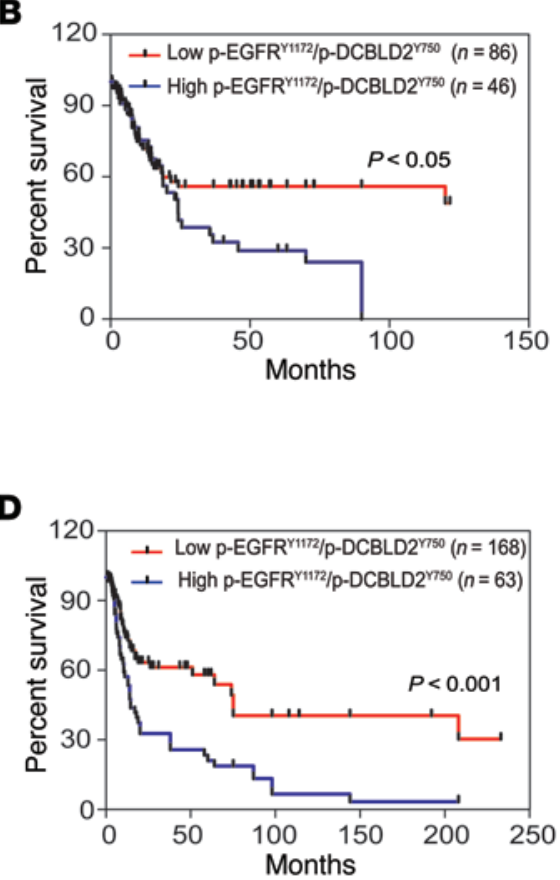

Figure 8. Coexpression of $\mathrm{p}-\mathrm{EGFR}^{\mathrm{v} 1172}$ and $p$-DCBLD2 ${ }^{\mathrm{V} 750}$ correlates with poorer prognoses of gliomas or HNCs. (A) A total of 132 clinical glioma specimens, including WHO grade II-IV gliomas, were analyzed by IHC. Representative images of serial sections of a GBM tissue using anti-p-EGFR ${ }^{\mathrm{V} 1172}$, anti-p-DCBLD2 ${ }^{\mathrm{\gamma 750}}$, anti-TRAF6, and anti-p-AKT T308 antibodies are shown. Insets: IgC controls of the same area in serial sections of these specimens. (B) Kaplan-Meier analyses of patients with high $\mathrm{p}-\mathrm{Y}$-EGFR ${ }^{\mathrm{Y} 1172} / \mathrm{p}-\mathrm{DCBLD2}{ }^{\mathrm{V} 750}$ expressing tumors (blue line) versus low p-EGFR ${ }^{\mathrm{V} 172} / \mathrm{p}-\mathrm{DCBLD2} 2^{\mathrm{V} 750}$-expressing tumors (red line) in IHC analyses in A. $P$ values were calculated by using log-rank test. Black bars indicate censored data. (C) A total of 231 clinical HNC specimens on tumor TMAs were analyzed by IHC staining. Representative images of serial sections of a HNC tissue. Insets: IgG controls of the same area in serial sections of these samples. (D) Kaplan-Meier analyses of patients with high p-EGFR ${ }^{\mathrm{V} 1172} / \mathrm{p}-\mathrm{DCBLD}{ }^{\mathrm{V} 750}$ expressing HNCs (blue line) versus low p-EGFR ${ }^{\mathrm{y} 1172} / \mathrm{p}-\mathrm{DCBLD}{ }^{\mathrm{\gamma} 750}$-expressing HNCs (red line) in IHC analyses. $P$ values were calculated by using log-rank test. Black bars indicate censored data. (E) A working model of EGFR/DCBLD2/ TRAF6/AKT signaling in tumorigenesis. Data in $\mathbf{A}$ and $\mathbf{C}$ are representative of 2 independent experiments. Scale bars: $50 \mu \mathrm{m}$. Arrows in $\mathbf{A}$ and $\mathbf{C}$ indicate positive staining.

mediates EGFR/EGFRvIII stimulation of p-DCBLD2 ${ }^{\mathrm{Y} 750} / \mathrm{AKT}$ signaling, promoting glioma tumorigenesis. To reconcile this discrepancy, we examined endogenous levels of DCBLD2, TRAF6, and SKP2 in cancer cell lines derived from breast cancers and gliomas. We found that DCBLD2 was expressed at low levels in 4 of 5 breast cancer cell lines that were used in our previous study (Supplemental Figure 15 and ref. 9), whereas high levels of DCBLD2 and TRAF6 were found in all 9 glioma cell lines examined (Supplemental Figure 2A and Supplemental Figure 15). Significantly, when compared with that in TRAF6 ${ }^{\text {WT }}$ mouse embryonic fibroblasts (MEFs), DCBLD2 was expressed at very low levels in TRAF6-deficient (Traf6 ${ }^{--}$) MEFs, in which endogenous TRAF6 was genetically depleted (Supplemental Figure 16A), thereby suggesting a correlation of endogenous levels of expression of TRAF6 with DCBLD2 in different types of cells. To further support these observations, we knocked down endogenous TRAF6 using siRNAs or different shRNAs in parental SNB19 and U87 cells as well as U87/EGFRvIII cells, in which EGFRvIII activates DCBLD2/TRAF6/AKT signaling. When endogenous TRAF6 is knocked down by siRNAs in SNB19, U87, and U87/EGFRvIII glioma cells, the endogenous levels of DCBLD2 were markedly attenuated in these glioma cells (Supplemental Figure 16, B and C). These results strongly suggest that the presence of TRAF6 may affect DCBLD2 expression in MEFs and glioma cells.

Next, we examined whether DCBLD2 mediates EGFRvIII stimulation of TRAF6/AKT signaling, enhancing GBM tumorigenesis. As shown in Figure 6C, in the presence or absence of a PI3K inhibitor, LY294002, knockdown of either DCBLD2 (shD2) or TRAF6 (shT6) markedly inhibited EGFRvIII-stimu- 
lated $\mathrm{p}-\mathrm{AKT}^{\mathrm{T} 308}$ and anchorage-independent colony formation in soft agar. To a similar extent, regardless of whether AKT was knocked down by shRNAs, depletion of either DCBLD2 (shD2) or TRAF6 (shT6) still significantly attenuated EGFRvIII-stimulated p-AKT ${ }^{\mathrm{T} 308}$ and colony formation by U87/vIII cells (Figure 6D). Conversely, expression of a constitutively active AKT (Myr.AKT) in U87/vIII cells, in which DCBLD2 or TRAF6 was depleted, rescued EGFRvIII-stimulated p-AKT ${ }^{\mathrm{T} 308}$ and anchorage-independent growth in soft agar (Figure 6E). These data validate the connection of the EGFRvIII/DCBLD2/TRAF6/AKT signaling, demonstrating that DCBLD2/TRAF6 signaling mediates EGFRvIII stimulation of AKT and glioma cell tumorigenesis.

Last, we used a phospho-mimic mutant of DCBLD2 ${ }^{\mathrm{D} 750}$ to corroborate that $\mathrm{p}-\mathrm{DCBLD} 2{ }^{\mathrm{Y} 750}$ mediates EGFRvIII stimulation of AKT and glioma cell tumorigenesis. We found that reexpression of an shRNA-resistant dominant activating DCBLD2 ${ }^{\mathrm{D} 750}$ mutant, but not DCBLD2 ${ }^{\mathrm{WT}}$ in U87/vIII cells in which DCBLD2 was knocked down by an shRNA (Supplemental Figure 17A), rescued the inhibition of an EGFR inhibitor, erlotinib, on EGFRvIII-activated p-AKT ${ }^{\mathrm{T} 308}$, association of DCBLD2 with TRAF6, and colony formation (Figure 7, A and B). Similarly, in patient-derived GSC83 cells that express endogenous EGFRvIII at high levels (Supplemental Figure 4), reexpression of the shRNA-resistant DCBLD2 ${ }^{\mathrm{D} 750}$ mutant, but not DCBLD2 ${ }^{\mathrm{WT}}$, in DCBLD2-depleted GSC83 cells (Supplemental Figure 17B) also rescued erlotinib inhibition of $\mathrm{p}-\mathrm{AKT}^{\mathrm{T} 308}$, association of DCBLD2 with TRAF6, formation of neurospheres, and cell proliferation in these GSCs (Figure 7, C and D). Taken together, these data establish the role of EGFR/p-DCBLD2 ${ }^{\mathrm{Y} 750} / \mathrm{TRAF} 6 / \mathrm{AKT}$ signaling in promoting glioma tumorigenesis in U87 and patient-derived GSCs that express exogenous or endogenous EGFRvIII, respectively.

Expression of $p-E G F R^{Y 1172}$ and $p-D C B L D 2^{Y 750}$ is associated with decreased survival of patients with gliomas or HNCs. Increased expression of EGFR, EGFRvIII, and p-AKT is closely associated with a poor prognosis for patients with malignant gliomas and HNCs (3, 35). To further define the clinical relevance of our findings in this study and our previous finding that TRAF6 enhanced $\mathrm{p}-\mathrm{AKT}^{\mathrm{T} 308}$ (8), we examined expression of p-EGFR ${ }^{\mathrm{Y} 1172}$ (36), p-DCBLD2 ${ }^{\mathrm{Y} 750}$, TRAF6, and $\mathrm{p}-\mathrm{AKT}^{\mathrm{T} 308}$ in clinical cancer samples. Using antibodies with validated specificities against these 4 proteins, we performed IHC analyses on serial sections of 132 clinical glioma specimens and tumor tissue microarrays (TMAs) of HNCs comprising a total of 231 clinical samples (Supplemental Figures 18 and 19 and ref. 37). In both glioma and HNC tissues, coexpression of p-DCBLD2 ${ }^{\mathrm{Y} 750}$, TRAF6, and p-AKT ${ }^{\mathrm{T} 308}$ was found in the majority of p-EGFR ${ }^{\mathrm{Y} 1172}$-positive tumors (Figure 8, A and C). IB analyses on separate and independent cohorts of 19 snap-frozen clinical GBM specimens and 15 clinical HNC samples and corresponding normal tissues also revealed coexpression of EGFR, p-DCBLD2 ${ }^{\mathrm{Y} 750}$, TRAF6, and p-AKT ${ }^{\mathrm{T} 308}$ in 6 of 8 EGFR-expressing GBM tissues and 5 of 7 EGFR-expressing HNC samples (Supplemental Figure 20). Spearman's rank correlation analysis, based on quantification of the IHC staining (26), showed that these correlations in both types of cancers were statistically significant (Supplemental Tables 1 and 2). Kaplan-Meier analyses of survival showed that high expression levels of $\mathrm{p}-\mathrm{EGFR}^{\mathrm{Y} 1172}$ or $\mathrm{p}-\mathrm{DCBLD} 2^{\mathrm{Y} 750}$ could serve as predictors of a worse prognosis for patients with gliomas (Supplemental Figure 21). Moreover, coexpression of $\mathrm{p}-\mathrm{EGFR}^{\mathrm{Y} 1172}$ and $\mathrm{p}-\mathrm{DCBLD} 2^{\mathrm{Y} 750}$ at high levels (Figure 8, B and D) or coexpression of p-EGFR ${ }^{\mathrm{Y} 1172}$ / TRAF6 at high levels correlated with significantly shorter survivals in patients with gliomas or HNCs (Supplemental Figures 21 and 22). Additionally, IHC staining using antibodies with previously validated specificities for EGFRvIII (26) and p-DCBLD2 ${ }^{\mathrm{Y} 750}$ on the same cohort of clinical gliomas as shown in Figure 8A also revealed an association of this coexpression with worse clinical outcomes for patients with gliomas (Supplemental Figure 23). The significance of increased expression of p-EGFR ${ }^{\mathrm{Y} 1172}$ and $\mathrm{p}-\mathrm{DCBLD} 2^{\mathrm{Y} 750}$ in glioma malignancy is further supported by the positive correlation of increased IHC staining for these 2 factors in WHO grades II-IV gliomas compared with normal brain tissues (Supplemental Figure 24). Taken together, these data support the role of EGFR/p-DC$\mathrm{BLD} 2{ }^{\mathrm{Y} 750} / \mathrm{TRAF} 6 / \mathrm{AKT}$ signaling in the pathophysiology, clinical progression, and aggressiveness of human gliomas and HNCs. These results also suggest that $\mathrm{p}-\mathrm{DCBLD} 2^{\mathrm{Y} 750}$ and EGFR could be useful clinical markers in the diagnosis and assessment of clinical outcomes in gliomas and HNCs.

\section{Discussion}

Aberrant activation of EGFR/AKT signaling frequently occurs in human cancers, including GBMs, HNCs, lung cancers, and melanomas, and promotes tumorigenesis, progression, invasion, and metastasis of these malignant tumors $(3,5,6,23)$. Typically, activation of oncogenic EGFR/AKT signaling in human cancers is caused by gene amplification, overexpression, or activating mutations of EGFR. To a larger extent, this is also a common genetic mechanism for other oncogenic RTKs, such as PDGFR and MET, and activated mutations of PI3K, a direct activator of AKT or loss of function of PTEN, a direct suppressor of AKT $(2,7,38)$. Moreover, in breast and prostate cancers, AKT can also be activated by other RTKs through TRAF6-mediated signaling $(8,9)$. In this study, we describe a novel function of DCBLD2, an orphan membrane receptor that has an unclear role in human cancers $(15-17,39)$, in mediating EGFR/AKT-driven tumorigenesis. We show that DCBLD2 is expressed at high levels in clinical samples of GBMs and HNCs. EGFR/EGFRvIII supports DCBLD2 interaction with TRAF6 through p-Y of Y750 in DCBLD2 within a consensus motif for TRAF6 binding. The association of $\mathrm{p}-\mathrm{DCBLD} 2{ }^{\mathrm{Y} 750}$ with TRAF6 activates oncogenic AKT signaling, thereby promoting tumorigenesis of GBMs, HNCs, lung cancers, and melanomas in vitro and in vivo (Figure $8 \mathrm{E}$ ). The clinical importance of our observations is strongly supported by the data showing that p-EGFR ${ }^{\mathrm{Y} 1172}$, p-DCBLD2 ${ }^{\mathrm{Y} 750}$, TRAF6, and p-AKT ${ }^{\mathrm{T} 308}$ are coexpressed in clinical gliomas and HNCs and coexpression of $\mathrm{p}-\mathrm{EGFR}^{\mathrm{Y} 1172}$ and $\mathrm{p}-\mathrm{DCBLD} 2^{\mathrm{Y} 750}$ correlates with shorter survival outcomes in patients with gliomas or HNCs. Thus, this study provides clinical and mechanistic evidence demonstrating that DCBLD2 upregulation is critical for EGFR-driven tumorigenesis in human cancers.

DCBLD2 was initially identified as an upregulated gene during vascular injury (13) and is a marker for vascular remodeling. In vascular smooth muscle cells (VSMCs), DCBLD2 suppresses PDGFR- $\beta$ signaling at surface levels of PDGFR through modulating expressions of c-CBL, an E3 ubiquitin ligase that ubiquitinates PDGFR- $\beta$, inhibiting VSMC growth. However, the downstream effectors that mediate DCBLD2 activation of c-CBL were 
not identified $(14,40)$. A recent study showed that, in endothelial cells (ECs), DCBLD2 enhances VEGFR-2 signaling through direct association with VEGFR-2, preventing VEGFR-2 complex formation with its negative regulator, namely VE-cadherin and protein tyrosine phosphatases (PTPs) PTP-1B and T cell-PTP (41). Thus, DCBLD2 acts as a positive regulator of VEGFR-2-promoted developmental and adult angiogenesis (41). In the present study, we described a distinct mechanism by which DCBLD2 mediates EGFR/EGFRvIII/AKT-driven tumorigenesis. In cancer cells that we examined, EGFR/EGFRvIII does not interact with DCBLD2 directly but generates a specific DCBLD2 ${ }^{\mathrm{Y} 750}$ phosphorylation within the TRAF6 interaction motif (TIM) that regulates the interaction of DCBLD2 with TRAF6. The DCBLD2/TRAF6 interaction increases E3 ligase activity of TRAF6 that in turn stimulates AKT-oncogenic signaling, leading to enhanced tumorigenic activity of cancer cell lines derived from glioma, lung cancer, HNC, and melanoma. This investigation identifies a previously unrecognized mechanism, in which DCBLD2/TRAF6 functions as a signaling node in mediating EGFR/EGFRvIII stimulation of oncogenic AKT signaling, thereby promoting tumorigenesis in human cancers. Additionally, our results and the aforementioned studies $(14,41)$ also provide excellent evidence demonstrating the context-dependent roles of DCBLD2 in modulating different RTK signaling that are each unique in VSMCs, ECs, and tumor cells.

DCBLD2 is a single membrane-spanning protein that it does not appear to contain any canonical signal module in its cytoplasmic domain (13). In this study, we identified and validated a major TIM in the $C$ terminus of the DCBLD2 protein, PAPDELVYQ (bold font represents consensus AA in the sequence) (29) at AA residues 743-751, that interacts with TRAF6. Significantly, EGFR/ EGFRvIII phosphorylates Y750 (p-Y750) within this region. The p-DCBLD2 ${ }^{\mathrm{Y} 750}$ is required for EGFR/EGFRvIII-driven tumorigenesis, interaction with TRAF6, and activation of TRAF6 E3 ligase activity important for AKT signaling. Structural analyses of the TIM (PxExAr/Ac; bold font represents consensus AA in the sequence) in several TRAF6-binding proteins, including CD40, TRANCE-R (RANK), and IRAK, showed that the Y residue of the sequence (referred to as position P3) may possess multiple conformations and is adjacent to several basic AA residues (29), suggesting the possibility that phosphorylation at this position may increase binding via either an electrostatic interaction or repulsion. Interestingly, mutation of the $\mathrm{P} 3$ residue of TRANCE-R into an alanine (A) abolished the interaction of TRAF 6 with its binding partners (29). Therefore, $\mathrm{p}$-Y750 could facilitate TRAF6 binding to DCBLD2, whereas mutation of Y750 into F750 would impair the binding. It is also possible that the presence of p-Y750 increases TRAF6 association with DCBLD2 by shifting the interaction motif in DCBLD2 into a highly exposed stage. Alternatively, unphosphorylated DCBLD2 might exist in a repressed conformation that is relieved by phosphorylation of Y750, thereby enhancing the interaction with TRAF6. Although beyond the scope of this study, investigation of these structural aspects would further illustrate the precise molecular mechanism of the enhanced TRAF6 interaction and facilitate the development of DCBLD2-targeted drugs.

Amplification and overexpression of genes are common genetic events during initiation and progression of human cancers (1). However, the imputed oncogenic properties of the amplified/overexpressed genes in tumorigenesis need to be supported by mechanistic and clinical studies. DCBLD2 is an understudied protein for its role in human diseases, including cancers. Here, we have shown that expression of DCBLD2 is increased in clinical GBMs and also demonstrated that DCBLD2 functions as a signal relay in mediating EGFR/EGFRvIII-driven tumorigenesis. Furthermore, our analyses of clinical gliomas and HNCs reveal a close correlation between coexpression of p-EGFR ${ }^{\mathrm{Y} 1172} / \mathrm{p}-\mathrm{DCBLD} 2^{\mathrm{Y} 750}$ and $\mathrm{p}-\mathrm{EGFR}^{\mathrm{Y} 1172} / \mathrm{TRAF} 6$ with poor prognoses in patients with gliomas or HNCs. These data not only reveal the importance of $\mathrm{p}-\mathrm{DCBLD} 2{ }^{\mathrm{Y} 750}$ and TRAF6 expression in the clinical prognosis of patients with gliomas or HNCs, but also validate the mechanistic data presented in this study, supporting that p-DCBLD2 ${ }^{\mathrm{Y} 750}$ and TRAF6 are involved in EGFR/ EGFRvIII/AKT-driven tumorigenesis in human cancers. Since genetic alterations of oncogenic RTKs and tumor suppressor genes stimulate tumor angiogenesis that is critical in cancer growth $(1,42,43)$, the context-dependent roles of DCBLD2 in modulating oncogenic EGFR/AKT signaling in tumor cells (this study), PDGFR- $\beta$ signaling in VSMCs (14), and VEGFR-2 stimulation in ECs (41) further demonstrate a comprehensive role of DCBLD2 in cancer progression and tumorigenesis, suggesting the necessity and feasibility of targeting DCBLD2 in different tumor compartments (tumor cells, VSMCs/pericytes, and ECs) in developing effective treatments for human cancers.

In conclusion, our findings identify DCBLD2 as a target of increased gene expression in clinical GBMs and HNCs and also demonstrate a previously unknown signal relay by which DCBLD2/TRAF6 mediates EGFR stimulation of AKT, thereby enhancing the oncogenic activity of the EGFR/AKT pathway in human cancers. The newly established roles of DCBLD2 and TRAF6 in EGFR-driven tumorigenesis provide a strong rationale for targeting these 2 signaling molecules in clinical treatment of human cancers with high levels of EGFR and DCBLD2 expression.

\section{Methods}

Cell lines. Glioma cells (U87, LN229, T98G, D54, LN444, LN443, LN340, and SNB19), human embryonic kidney cells (HEK293T), short-term cultured primary human GBM cells (GBM6, GBM14, and GBM39), HNC cells (PCI-15B, UM22A, Cal-33, and OSC-19), and breast cancer cells (MDA-MB-231, MDA-MB-468, and SKBr3) were maintained in Dulbecco's modified Eagle's medium (DMEM) supplemented with $10 \%$ fetal bovine serum and $1 \%$ penicillin and streptomycin. BT474 and SUM149 breast cancer cells were maintained in Ham's medium supplemented with $5 \%$ fetal bovine serum. A375, 16082, TPF-11-174, and UACC903 melanoma cells were maintained in Roswell Park Memorial Institute medium supplemented with $10 \%$ fetal bovine serum. A549, 201T, 343T, H23, H1650, and H3255 lung cancer cells were maintained in Basal Medium Eagle (BME) supplemented with $10 \%$ fetal bovine serum. U87, SNB19, and LN444 cell lines were also authenticated recently using a STR DNA fingerprinting at RADIL. U87/EGFRvIII and SNB19/EGFRvIII cell lines that overexpress exogenous EGFRvIII were established and characterized as previously described $(25,26)$.

Seven patient-derived GSC lines that were recently characterized were used in this study: JK018, JK042, JK083, JK092 (28), proneural GSC 528, and mesenchymal GSC83 and GSC1123 (27). Patient- 
derived GSCs were cultured in DMEM/F12 (Invitrogen), supplemented with B27 (2\%, Invitrogen), penicillin and streptomycin (1\%, Invitrogen), Heparin (5 $\mu \mathrm{g} / \mathrm{ml}$, Sigma-Aldrich), EGF $(20 \mathrm{ng} / \mathrm{ml})$, and basic FGF (20 ng/ml, Peprotech) and grown in suspension plates or flasks with filter caps. Cells were expanded by changing half of the cell culture medium at least every 2 days. Cells were passaged by pelleting the cells with low-speed centrifugation $(200 \mathrm{~g}$ for 2 minutes), removing supernatant, dissociating the pellet using gentile mechanical up-and-down pipetting, and, if needed, enzymatic dissociation with StemPro Accutase (1 ml, Invitrogen). Cell lines were cultured in water-jacketed humidity-controlled incubators at $37^{\circ} \mathrm{C}$ and $5 \% \mathrm{CO}_{2}$. Cell transfections or infections were performed as previously described $(26,44)$.

Antibodies and reagents. The following antibodies were used in this study: anti-TRAF6 (H-274), anti-TRAF6 (D-10), anti-SKP2 (H-435), anti-Met (C-12), anti-phospho-PDGFR $\alpha$ (sc-12911, Y754), and anti- $\beta$-actin (I-19) antibodies (Santa Cruz Biotechnology); an anti-Ub antibody (BD Transduction Laboratories); an anti-DCBLD2 antibody (Sigma-Aldrich); a monoclonal anti-Flag M2 antibody (Sigma-Aldrich); anti-phospho-p44/42 MAP Kinase (Thr202/Tyr204, no. 9101), anti-p44/42 MAP Kinase (no. 9102), anti-phospho-AKT (Ser473, no. 4060, and Thr308, no. 2965), anti-AKT (no. 9272), and anti-phospho-EGFR (Y1045) antibodies (Cell Signaling Technology); an anti-phospho-EGFR (Y1172) antibody (Signalway Antibody); an anti-phospho-c-Met antibody (pY1230/1234/1235; BioSource International); an anti-Ki67 antigen (NCL-Ki67p) antibody (Leica Microsystems Inc.); an anti-pan-phosphotyrosine (4G10) antibody (Millipore-Upstate); an anti-EGFR antibody (Ab-1; Oncogene Science), and a mouse monoclonal anti-EGFR antibody (clone EGFR-113 for IHC, Vector Laboratories) (37). An anti-EGFRvIII-specific antibody DH8.3 was previously characterized (26). A rabbit polyclonal antiphospho-DCBLD2 ${ }^{\mathrm{Y} 750}$ antibody was produced by immunizing animals with a synthetic phospho-peptide corresponding to residues surrounding Y750 of human DCBLD2 (Pacific Immunology). The antibodies were then affinity purified. The secondary antibodies were from Vector Laboratories or Jackson ImmunoResearch Laboratories. Peroxidase-blocking reagent was from DAKO. AquaBlock was from East Coast Biologics Inc. Erlotinib was from LC Laboratories. Cell culture media and other reagents were from Invitrogen, Sigma-Aldrich, VWR, or Thermo Fisher Scientific.

Plasmids. DCBLD2 cDNA was amplified by PCR (5' CGGCGCGCCATGGCGAGCCGGGCGGTG $3^{\prime}$ and $5^{\prime}$ GGACGGTCCGTGAAGGATTTCTTTAAAAAC $3^{\prime}$ ) and then inserted into pCMV6-Flag-Myc vector with Asc I and Rsr II digestion. pMXI-Flag-Myc-DCBLD2 was derived from pCMV6-Flag-Myc-DCBLD2. A pcDNA3-3xHA-TRAF6 was derived from pcDNA3-TRAF6-YFP by reverse transcription PCR (RT-PCR) as previously described $(26,44)$. A pcDNA3-EGFRvIII and pMT107-His-Ub were described in our previous studies $(8,26)$. $\mathrm{TRAF}^{670 \mathrm{~A}}, \mathrm{DCBLD}^{\mathrm{F} 621}, \mathrm{DCBLD} 2^{\mathrm{F} 750}, \mathrm{DCBLD}^{\mathrm{F} 621 / \mathrm{F} 750}, \mathrm{DCBLD}^{\mathrm{D} 750}$, DCBLD2 $2^{\mathrm{P} 641 \mathrm{Q}}$, and $\mathrm{DCBLD} 2^{\mathrm{P} 74 \mathrm{Q}}$ point mutations were generated using a QuikChange Multi Site-Directed Mutagenesis Kit (Agilent Technologies) following the manufacturer's protocol. GFP shRNA, DCBLD2 shRNAs, AKT1/2 siRNAs, and control siRNA were purchased from Dharmacon, Thermo Fisher Scientific. The pSuper-GFP-TRAF6 shRNA no. 1 and shRNA no. 2 were generated as previously described $(26,44)$. HA-AKT and Myr-AKT (constitutively active form) in a pBabe retroviral vector were from Addgene.
Digital karyotyping. Digital karyotyping was performed on 10 GBM samples. Protocols for extraction of DNA and analysis of samples can be found in a previously published study (45). Experimental tag sequences were visualized using SageGenie DKView (http://cgap.nci.nih.gov/ SAGE/DKViewHome). Candidate genes were identified using the University of California, Santa Cruz Genome Browser, assembly hg16.

SAGE. Analysis of SAGE data was performed on data available via SAGE Genie (http://cgap.nci.nih.gov/SAGE). Analysis for significance between 2 groups was completed using a 2-tailed $t$ test with Welch correction.

Fluorescence in situ hybridization. Fluorescence in situ hybridization was performed as previously described (45). Probes for DCBLD2 and a control chromosome 3 reference were generated using bacterial artificial chromosome clones (Invitrogen) RP1179M2 (123,351,114-123,538,059 Mb) and RP11-297J9 (168,937,657$169,120,179 \mathrm{Mb})$, respectively.

TCGA data analysis. Gene expression and genomic alterations were analyzed across GBM subtypes. Data were acquired from the TCGA data portal. GBM subtype classification was based on Verhaak et al. (22). Copy number was analyzed via the genome-wide SNP_6 $\log _{2}$ ratio. Gene expression is shown as the Agilent G4502A_07 $\log _{2}$ tumor/ normal ratio, representing the ratio of tumor to normal expression, with the expression value presented as the $\log _{2}$ of the ratio of tumor expression compared with a synthetic normal sample.

$R T-P C R$ and Q-PCR. RT-PCR were performed as previous described using forward primer (5'-gagtcgggctctggaggaaaag- $\left.3^{\prime}\right)$ and reverse primer (5'-gatccagaggaggagtatgtgtg-3') that specifically distinguish gene transcripts of EGFR WT or EGFRvIII (46) in various patient-derived GSCs. An approximately 1,100-bp PCR product indicates expression of WT EGFR, while an approximately 300-bp PCR product reveals EGFRvIII expression in these cells. Gene expression was analyzed via quantitative real-time PCR, as previously described (45), using the Applied Biosciences 7900HT Fast Real-Time PCR System. Gene expression was normalized to GAPDH. Q-PCR was performed in triplicate. Threshold cycle numbers were calculated using Applied Biosystems SDS software 2.2.2.

In vitro EGFR phosphorylation. In vitro EGFR phosphorylation of DCBLD2 was determined as previously described (44). Briefly, FlagDCBLD2 ${ }^{\mathrm{WT}}$ or Flag-DCBLD2 ${ }^{\mathrm{F} 750}$ cDNAs were separately transfected into HEK293T cells for 48 hours. Cells were then lysed, and FlagDCBLD2 ${ }^{\mathrm{WT}}$ or Flag-DCBLD2 $2^{\mathrm{F} 750}$ proteins were subjected to IP using an anti-Flag antibody. The precipitates were then treated with $15 \mu \mathrm{M}$ of a recombinant YOP protein phosphatase (PTP, Enzo Life Science) at $30^{\circ} \mathrm{C}$ for 1 hour in $1 \mathrm{X}$ YOP reaction buffer $(50 \mathrm{mM}$ citrate, $\mathrm{pH}$ 6.0, $100 \mathrm{mM} \mathrm{NaCl}, 1 \mathrm{mM}$ EDTA, and $1 \mathrm{mM}$ DTT) containing $1 \mathrm{mg} / \mathrm{ml} \mathrm{BSA}$, washed 3 times with $\mathrm{PBS}$, and incubated with or without a recombinant active EGFR (Active Motif) at $30^{\circ} \mathrm{C}$ for 30 minutes. The reaction products were mixed with an equal volume of IP buffer or $2 X$ SDS sample buffer and examined by IP and IB analyses.

$I P$ and IB. IB and IP analyses were performed as previously described (44).

Cell proliferation and viability assays. As previously described (44), in vitro cell proliferation analyses were performed using a WST1 Assay Kit (Roche), and cell viability assays were performed using a TUNEL Assay Kit (Roche).

Colony formation assay. Soft agar colony formation assay was performed as previously described (47). 
siRNA and shRNA knockdown, transient transfection, and reexpression of shRNA-resistant DCBLD2 $2^{W T}$ and various mutants. These assays were performed as previously described (44).

Analyses of neural sphere sizes and GSC counting. Analyses of neural sphere sizes and GSC counting were performed as we recently described (27). Briefly, 200, 100, and 50 cells were separately sorted into each well of 96 -well plates in at least 8 replicates by a BD FACSAria III flow cytometer and then cultured in the GSC medium in the presence of erlotinib (an EGFR inhibitor) or DMSO as control for 6 days. Sphere size was then observed at day 6 under an inverted microscope from Nikon equipped with a digital camera.

For GSC counting, 10,000 cells per well were sorted into a 96-well plate in at least 8 replicates by a BD FACSAria III flow cytometer and then cultured in the GSC medium in the presence of erlotinib or DMSO as control for 6 days. Single cells were dissociated from neurospheres with StemPro Accutase. The cell number for living GSCs was counted under an inverted microscope using a hematocytometer following the addition of 50\% (vol/vol) Trypan Blue (Invitrogen).

Tumorigenesis studies. Athymic $(\mathrm{Ncr} n u / n u)$ female mice at an age of 6 to 8 weeks (Taconic Farms) were used for all animal experiments. Human glioma cells $\left(5 \times 10^{5}\right.$ cells in $\left.5 \mu \mathrm{l} \mathrm{PBS}\right)$ or patientderived GSCs $\left(5 \times 10^{4}\right.$ cells in $2 \mu \mathrm{l}$ PBS $)$ were stereotactically implanted into the brains of individual mice, with 5 mice per group. The glioma-bearing mice were sacrificed 2 or 5 weeks after implantation. The brains were removed, processed, and analyzed as previously described (44).

IHC of human and mouse glioma specimens. The tissue sections from paraffin-embedded deidentified human glioma and HNC specimens were stained with antibodies against p-DCBLD2 $2^{\mathrm{Y} 750}$ (1:100), EGFR (clone EGFR-113, 1:10), p-EGFR ${ }^{\mathrm{Y} 1172}$ (1:50), EGFRvIII (clone 8.3, 1:50), TRAF6 (H-274, 1:100), and p-AKT ${ }^{\mathrm{T} 308}$ (no. 2963, 1:100). Nonspecific IgGs were used as negative controls. In total, 132 primary human glioma specimens and 4 normal brain tissues without notable pathological lesions or history were collected from 2001 to 2008 at Saitama Medical University and Kyorin University. Human glioma samples include 31 WHO grade II, 23 grade III, and 78 grade IV glioma tumors. 232 primary human HNC specimens were collected from 1992 to 2012 and spotted onto glass slides as TMAs in triplicates per tumor sample at University of Pittsburgh, Pittsburgh, Pennsylvania, USA. These clinical cancer specimens were examined and diagnosed by pathologists at Saitama Medical University, Kyorin University, or University of Pittsburgh, respectively. IHC staining for EGFR using the anti-EGFR antibody was first performed in all 132 glioma samples. Forty-six glioma specimens showed EGFR protein expression at high levels (signal strengths at $2+$ or $3+$ ). These 46 glioma tumor samples were then stained with the anti-p-EGFR ${ }^{\mathrm{Y} 1172}$ antibody. For HNC TMAs, IHC staining was performed separately using anti-EGFR or anti-p-EGFR ${ }^{\mathrm{V} 1172}$ antibodies. IHC staining was quantified as we previously described (44): $3+$, signals in $\sim 50 \%$ tumor cells; $2+$, signals in $~ 25 \%$ tumor cells; $1+$, signals in $\sim 5 \%$ to $25 \%$ tumor cells; \pm , low or no signals in $<1 \%$ tumor cells; -, no detectable signals in all tumor cells (0\%). Tumors with - or \pm staining were considered as low expressing, and tumors with $1+$ to $3+$ scores were considered high expressing. Analyses of Spearman's rank correlation and Kaplan-Meier survival were performed as previously described (26).
Mouse brain sections with various tumors were analyzed by IHC using an anti-Ki-67 antibody (1:200) or a TUNEL Staining Kit (Roche). Images were captured using an Olympus BX53 microscope equipped with an Olympus DP72 digital camera. Five random images per section of mouse brain were obtained, and the percentage of Ki-67- or TUNEL-positive cells was quantified and statistically analyzed as previously described (44).

Statistics. GraphPad Prism version 5.0 for Windows was used to perform 1-way ANOVA with Newman-Keuls post-hoc test or paired 2-tailed Student's $t$ test as previously described (44). $P$ values of less than 0.05 were considered significant.

Study approval. All the work related to human tissues was performed at the University of Pittsburgh, Northwestern University, Duke University, and The Ohio State University under institutional review board-approved protocols, according to NIH guidelines. All experiments using animals were performed at the University of Pittsburgh and Northwestern University under the Institutional Animal Care and Use Committee-approved protocols, according to NIH guidelines.

\section{Acknowledgments}

We thank E. Van Meir, Y. Zhou, J.N. Sarkaria, G.P. Robertson, J. Kirkwoord, and J.M. Siegfried for providing reagents and C. Di, Y. Yoo, and J.L. May for help in some of the experiments. This work was supported by a Zell Scholar Award from the Zell Family Foundation, funds of Northwestern Brain Tumor Institute and Department of Neurology at Northwestern University Feinberg School of Medicine, and NIH grants R01CA130966, R01CA158911, and R01CA158911S1 (to S.Y. Cheng); Brain Cancer Research Awards from the James S. McDonnell Foundation (to B. Hu, F.B. Furnari, and H. Yan); Duquesne University Hunkele Dreaded Disease Award and the Interleukin Foundation (to P.E. Auron); NIH P50 CA097190 and the American Cancer Society (to J.R. Grandis); NIH T32 DC000066 (to M.L. Hedberg); American Cancer Society MRSG-08-108-01 and NIHP01 CA163205, R21CA175875, R01NS083767, and R01NS087913 (to I. Nakano), the Michael J. Marchese Endowed Chair in Neurological Surgery at Northwestern University (to A.T. Parsa); NIH R01CA136787 and R01CA149321 (to H.K. Lin); NIH R01CA140316, ACS RSG-10126-01-CCE, a Pediatric Brain Tumor Foundation Institute grant, a Voices Against Brain Cancer Foundation grant, The V Foundation, and an Accelerate Brain Cancer Cure Foundation grant (to H. Yan); NIH R01NS080939 (to F.B. Furnari); The Defeat GBM Research Collaborative, a subsidiary of National Brain Tumor Society (to W.K. Cavenee and F.B. Furnari); the Chinese Ministry of Science and Technology (2012CB966800), the National Natural Science Foundation of China (81130038 and 81372189), Key Discipline and Specialty Foundation of Shanghai Health Bureau, and KC Wong Foundation (to W.-Q. Gao); and National Natural Science Foundation of China (no. 81372704), Innovation Program of Shanghai Municipal Education Commission in China (no. 14ZZ111), the Program for Professor of Special Appointment (Eastern Scholar) at Shanghai Institutions of Higher Learning, and the State Key Laboratory of Oncogenes and Related Genes in China (no. 90-14- 01) (to H. Feng). S.Y. Cheng is a Zell Scholar at Northwestern University and W.K. Cavenee is a fellow of the National Foundation for Cancer Research. 
Address correspondence to: Shi-Yuan Cheng or Bo Hu, Department of Neurology and Northwestern Brain Tumor Institute, Center for Genetic Medicine, The Robert H. Lurie Comprehensive Cancer Center, Northwestern University Feinberg School of Medicine, 303 E Chicago Ave., Chicago, Illinois 60611, USA. Phone: 312.503.3043; E-mail: shiyuan.cheng@northwestern. edu (S.Y. Cheng), bo.hu@northwestern.edu (B. Hu). Or to: Hai Yan, The Preston Robert Tisch Brain Tumor Center, Pediatric Brain Tumor Foundation Institute at Duke, and Department of Pathology, Duke University Medical Center, DUMC Box 3624,
Durham, North Carolina 27710, USA. Phone: 919.668.7850; E-mail: hai.yan@dm.duke.edu.

Giselle Y. Lopez's present address is: Department of Pathology, UCSF, San Francisco, California, USA.

Christopher G. Duncan's present address is: Department of Urology, Department of Biochemistry and Molecular Biology, Norris Comprehensive Cancer Center, Keck School of Medicine, USC, Los Angeles, California, USA.
1. Hanahan D, Weinberg RA. Hallmarks of cancer: the next generation. Cell. 2011;144(5):646-674.

2. Cancer Genome Atlas Research Network. Comprehensive genomic characterization defines human glioblastoma genes and core pathways. Nature. 2008;455(7216):1061-1068.

3. Leemans CR, Braakhuis BJ, Brakenhoff RH. The molecular biology of head and neck cancer. Nat Rev Cancer. 2011;11(1):9-22.

4. Ohashi K, Maruvka YE, Michor F, Pao W. Epidermal growth factor receptor tyrosine kinase inhibitor-resistant disease. J Clin Oncol. 2013;31(8):1070-1080.

5. Sharafinski ME, Ferris RL, Ferrone S, Grandis JR. Epidermal growth factor receptor targeted therapy of squamous cell carcinoma of the head and neck. Head Neck. 2010;32(10):1412-1421.

6. Dunn GP, et al. Emerging insights into the molecular and cellular basis of glioblastoma. Genes Dev. 2012;26(8):756-784.

7. Klein S, Levitzki A. Targeting the EGFR and the PKB pathway in cancer. Curr Opin Cell Biol. 2009;21(2):185-193.

8. Yang WL, et al. The E3 ligase TRAF6 regulates Akt ubiquitination and activation. Science. 2009;325(5944):1134-1138.

9. Chan $\mathrm{CH}$, et al. The Skp2-SCF E3 ligase regulates Akt ubiquitination, glycolysis, herceptin sensitivity, and tumorigenesis. Cell. 2012;149(5):1098-1111.

10. Wang KZ, Galson DL, Auron PE. TRAF6 is autoinhibited by an intramolecular interaction which is counteracted by trans-ubiquitination. J Cell Biochem. 2010;110(3):763-771.

11. Wang KZQ, et al. TRAF6 activation of PI 3-kinase-dependent cytoskeletal changes is cooperative with Ras and is mediated by an interaction with cytoplasmic Src. J Cell Sci. 2006;119(pt 8):1579-1591.

12. Liu A, et al. TRAF6 protein couples Toll-like receptor 4 signaling to Src family kinase activation and opening of paracellular pathway in human lung microvascular endothelia. J Biol Chem. 2012;287(20):16132-16145.

13. Kobuke K, et al. ESDN, a novel neuropilin-like membrane protein cloned from vascular cells with the longest secretory signal sequence among eukaryotes, is up-regulated after vascular injury. J Biol Chem. 2001;276(36):34105-34114.

14. Guo X, Nie L, Esmailzadeh L, Zhang J, Bender JR, Sadeghi MM. Endothelial and smooth muscle-derived neuropilin-like protein regulates platelet-derived growth factor signaling in human vascular smooth muscle cells by modulating receptor ubiquitination. J Biol Chem.
2009;284(43):29376-29382.

15. Koshikawa K, et al. Significant up-regulation of a novel gene, CLCP1, in a highly metastatic lung cancer subline as well as in lung cancers in vivo. Oncogene. 2002;21(18):2822-2828.

16. Hofsli E, Wheeler TE, Langaas M, Laegreid A, Thommesen L. Identification of novel neuroendocrine-specific tumour genes. Br JCancer. 2008;99(8):1330-1339.

17. Kim M, et al. Epigenetic down-regulation and suppressive role of DCBLD2 in gastric cancer cell proliferation and invasion. Mol Cancer Res. 2008;6(2):222-230.

18. Pasaje CF, et al. DCBLD2 gene variations correlate with nasal polyposis in Korean asthma patients. Lung. 2012;190(2):199-207.

19. Chen Y, et al. Phosphoproteomics identified Endofin, DCBLD2, and KIAA0582 as novel tyrosine phosphorylation targets of EGF signaling and Iressa in human cancer cells. Proteomics. 2007;7(14):2384-2397.

20. Huang PH, et al. Quantitative analysis of EGFRvIII cellular signaling networks reveals a combinatoria therapeutic strategy for glioblastoma. Proc Natl Acad Sci U S A. 2007;104(31):12867-12872.

21. Guo A, et al. Signaling networks assembled by oncogenic EGFR and c-Met. Proc Natl Acad Sci US A. 2008;105(2):692-697.

22. Verhaak RG, et al. Integrated genomic analysis identifies clinically relevant subtypes of glioblastoma characterized by abnormalities in PDGFRA, IDH1, EGFR, and NF1. Cancer Cell. 2010;17(1):98-110.

23. Seshacharyulu P, Ponnusamy MP, Haridas D, Jain M, Ganti AK, Batra SK. Targeting the EGFR signaling pathway in cancer therapy. Expert Opin Ther Targets. 2012;16(1):15-31.

24. Wykosky J, Fenton T, Furnari F, Cavenee WK. Therapeutic targeting of epidermal growth factor receptor in human cancer: successes and limitations. Chin J Cancer. 2011;30(1):5-12.

25. Nishikawa R, et al. A mutant epidermal growth factor receptor common in human glioma confers enhanced tumorigenicity. Proc Natl Acad Sci US A. 1994;91(16):7727-7731.

26. Feng $\mathrm{H}$, et al. Phosphorylation of dedicator of cytokinesis 1 (Dock180) at tyrosine residue Y722 by Src family kinases mediates EGFRvIII-driven glioblastoma tumorigenesis. Proc Natl Acad Sci U S A. 2012;109(8):3018-3023.

27. Mao P, et al. Mesenchymal glioma stem cells are maintained by activated glycolytic metabolism involving aldehyde dehydrogenase 1A3. Proc Natl Acad Sci U S A. 2013;110(21):8644-8649.
28. Srikanth M, Das S, Berns EJ, Kim J, Stupp SI, Kessler JA. Nanofiber-mediated inhibition of focal adhesion kinase sensitizes glioma stemlike cells to epidermal growth factor receptor inhibition. Neuro Oncol. 2013;15(3):319-329.

29. Ye H, et al. Distinct molecular mechanism for initiating TRAF6 signalling. Nature. 2002;418(6896):443-447.

30. Giannini C, et al. Patient tumor EGFR and PDGFRA gene amplifications retained in an invasive intracranial xenograft model of glioblastoma multiforme. Neuro Oncol. 2005;7(2):164-176.

31. Yiin JJ, et al. ZD6474, a multitargeted inhibitor for receptor tyrosine kinases, suppresses growth of gliomas expressing an epidermal growth factor receptor mutant, EGFRvIII, in the brain. $\mathrm{Mol}$ Cancer Ther. 2010;9(4):929-941.

32. Starczynowski DT, et al. TRAF6 is an amplified oncogene bridging the RAS and NF- $\mathrm{KB}$ pathways in human lung cancer. J Clin Invest. 2011;121(10):4095-4105.

33. Zhong L, Cao F, You Q. Effect of TRAF6 on the biological behavior of human lung adenocarcinoma cell. Tumour Biol. 2013;34(1):231-239.

34. Peng Z, Shuangzhu Y, Yongjie J, Xinjun Z, Ying L. TNF receptor-associated factor 6 regulates proliferation, apoptosis, and invasion of glioma cells. Mol Cell Biochem. 2013;377(1-2):87-96.

35. Van Meir EG, Hadjipanayis CG, Norden AD, Shu HK, Wen PY, Olson JJ. Exciting new advances in neuro-oncology: the avenue to a cure for malignant glioma. CA Cancer J Clin. 2010;60(3):166-193.

36. Yang W, et al. ERK1/2-dependent phosphorylation and nuclear translocation of PKM2 promotes the Warburg effect. Nat Cell Biol. 2012;14(12):1295-1304.

37. Biernat W, Huang H, Yokoo H, Kleihues P, Ohgaki H. Predominant expression of mutant EGFR (EGFRvIII) is rare in primary glioblastomas. Brain Pathol. 2004;14(2):131-136.

38. Liu KW, Hu B, Cheng SY. Platelet-derived growth factor receptor alpha in glioma: a bad seed. Chin J Cancer. 2011;30(9):590-602.

39. Pagnotta SM, et al. Ensemble of gene signatures identifies novel biomarkers in colorectal cancer activated through PPAR $\gamma$ and TNF $\alpha$ signaling. PLoS One. 2013;8(8):e72638.

40. Sadeghi MM, et al. ESDN is a marker of vascular remodeling and regulator of cell proliferation in graft arteriosclerosis. Am J Transplant. 2007;7(9):2098-2105.

41. Nie L, et al. Transmembrane protein ESDN promotes endothelial VEGF signaling and regulates angiogenesis. JClin Invest. 2013;123(12):5082-5097. 
42. Zerrouqi A, Pyrzynska B, Febbraio M, Brat DJ, Van Meir EG. P14ARF inhibits human glioblastoma-induced angiogenesis by upregulating the expression of TIMP3. JClin Invest. 2012;122(4):1283-1295.

43. Welti J, Loges S, Dimmeler S, Carmeliet P. Recent molecular discoveries in angiogenesis and antiangiogenic therapies in cancer. J Clin Invest. 2013;123(8):3190-3200.
44. Feng H, et al. Activation of Rac1 by Src-dependent phosphorylation of Dock180(Y1811) mediates PDGFR $\alpha$-stimulated glioma tumorigenesis in mice and humans. JClin Invest. 2011;121(12):4670-4684.

45. Wang TL, et al. Digital karyotyping identifies thymidylate synthase amplification as a mechanism of resistance to 5-fluorouracil in metastatic colorectal cancer patients. Proc Natl Acad Sci U S A.
2004;101(9):3089-3094.

46. Inda MM, et al. Tumor heterogeneity is an active process maintained by a mutant EGFR-induced cytokine circuit in glioblastoma. Genes Dev. 2010;24(16):1731-1745.

47. Liu KW, et al. SHP-2/PTPN11 mediates gliomagenesis driven by PDGFRA and INK4A/ARF aberrations in mice and humans. JClin Invest. 2011;121(3):905-917. 